\title{
A MIXED FINITE ELEMENT METHOD FOR EWOD THAT DIRECTLY COMPUTES THE POSITION OF THE MOVING INTERFACE
}

\author{
RICHARD S. FALK AND SHAWN W. WALKER
}

\begin{abstract}
A new mixed finite element method is proposed and analyzed for simulating two-phase droplet motion in a micro-scale device driven by Electrowetting-On-Dielectric (EWOD). The new feature of the method is that the finite element scheme is based on a weak formulation of the problem which includes the position of the moving interface and the curvature of its boundary as basic unknowns to be determined along with the velocity field and pressure. Well-posedness of the semi-discrete and fully discrete formulations and error estimates with minimal regularity assumptions are proved. Numerical examples are given to illustrate the robustness of the method.
\end{abstract}

\section{INTRODUCTION}

This paper presents a new mixed finite element method to simulate two-phase droplet motion in a microscale device driven by Electrowetting-On-Dielectric (EWOD) $[27,11,34,4,23]$. The device is essentially a Hele-Shaw cell (i.e., two closely spaced parallel plates) with a liquid droplet bridging the two plates. A grid of electrodes is embedded in the bottom plate which allows one to (locally) modify the surface tension by applying voltages to the electrodes. Thus, the droplet can move, split, and merge in a controlled fashion. Several applications use EWOD as a main driving force: mass spectrometry [44, 25], 'lab-on-a-chip' [33, 18, 20, 32], particle separation/concentration control [10, 41], auto-focus cell phone lenses [5], and colored oil pixels for laptops and video-speed smart paper [19, 30,31].

The two-dimensional mathematical model we consider is similar to the Hele-Shaw flow model with a modification of the boundary condition to account for the electrical surface tension effect. Unlike the work in $[42,43,40]$, we do not consider contact line frictional effects. In our model, we use an explicit front-tracking technique to capture interface motion. This is achieved by building the time-discrete update equation for the interface's position into the weak formulation as a linear constraint, which induces curvature to act as a Lagrange multiplier. To the best of our knowledge, this is novel in the context of moving interface problems. Many computational models exist for simulating the full electrowetting problem: $[3,24,21,7]$. In particular, [21] assumes quasi-static behavior of the droplet. A diffuse interface model is presented in [22] that simulates droplet motion in a scaled up version of the EWOD device. A Volume of Fluid (VoF) technique is used in $[3,24]$ to track droplet motion, but this method does not give precise information about the liquid-gas interface shape. A phase-field model (coupled to the Navier-Stokes fluid equations) has been proposed in $[14,1,16]$ which includes a new formulation of the problem and a detailed analysis of existence of solutions for the continuous time-dependant problem. Recently in [28], another diffuse interface model (also coupled to Navier-Stokes) is described that can include a wide range of custom contact line pinning models. In addition, existence of time-dependent solutions and a well-posed numerical scheme that is built on a projection scheme for the Navier-Stokes equations are presented.

An outline of the paper is as follows. In the next section, we describe the governing equations for the EWOD model. In Section 3, we discuss the procedure for time discretization. A variational formulation

Date: December 31, 2012.

2000 Mathematics Subject Classification. Primary: 65N30, Secondary: 76T10.

The work of the first author was supported in part by NSF grant DMS-0910540 and the work of the second author by NSF grant DMS-1115636. 
and well-posedness of the discrete time problem is given in Section 4 followed by a variational formulation and well-posedness of a fully discrete approximation scheme in Section 5. Section 6 contains error estimates. A discussion of the solution of the discrete equations is given in Section 7 followed by some numerical computations demonstrating the performance of the method in Section 8.

\section{THE EWOD MODEL}

Given an electrowetting forcing term $E=E(\boldsymbol{x})$, we seek to determine the velocity field $\boldsymbol{u}$ and pressure field $p$ satisfying

$$
\begin{gathered}
\alpha \partial_{t} \boldsymbol{u}+\beta \boldsymbol{u}+\nabla p=0, \quad \text { in } \Omega(t), \\
\operatorname{div} \boldsymbol{u}=0, \quad \text { in } \Omega(t), \\
p=\kappa+E, \quad \text { on } \Gamma(t)=\partial \Omega(t),
\end{gathered}
$$

where $\kappa$ is the curvature of $\Gamma(t)$, the droplet boundary representing the liquid-gas interface, and the nondimensional constants $\alpha$ and $\beta$ depend on fluid parameters and device geometry.

To complete the model, we need to describe how the boundary $\Gamma(t)$ changes with time. We have the following equation of motion for the time-varying liquid gas interface $\Gamma(t)$.

$$
\boldsymbol{X}(\cdot, t)=\Gamma(t), \quad \frac{d \boldsymbol{X}(s, t)}{d t}=[\boldsymbol{u}(\boldsymbol{X}(s, t), t) \cdot \boldsymbol{n}(\boldsymbol{X}(s, t), t)] \boldsymbol{n}(\boldsymbol{X}(s, t), t),
$$

where $\boldsymbol{X}(\cdot, t): I \rightarrow \Gamma(t) \subset \mathbb{R}^{2}$ is a parametrization of $\Gamma(t), s$ is the parametrization variable, $I$ the parametrization interval, $\boldsymbol{n}$ the outer unit normal vector of $\Omega$, and $\boldsymbol{t}$ the unit tangent vector along $\Gamma$. Finally, we recall an equation relating $\boldsymbol{X}(\cdot, t)$ to the vector curvature $\kappa \boldsymbol{n}$ of $\Gamma(t)$ :

$$
-\Delta_{\Gamma} \boldsymbol{X}=\kappa \boldsymbol{n}
$$

where $\Delta_{\Gamma}$ is the Laplace-Beltrami operator. Note: $\Delta_{\Gamma} \equiv \partial_{s}^{2}$, where $\partial_{s}$ is the derivative with respect to arc-length, when $\Gamma$ is a one-dimensional curve.

\section{Time Discretization}

We partition the time axis into time intervals $\Delta t_{i}$ and let $\Omega^{i}$ and $\Gamma^{i}$ be approximations to the domain $\Omega\left(t_{i}\right)$ and boundary interface $\Gamma\left(t_{i}\right)$ at time $t_{i}$. We further denote by $\boldsymbol{X}^{i}(\cdot)$ a parametrization of $\Gamma^{i}$.

3.1. Discrete Time Model. We obtain $\boldsymbol{u}^{i+1}$, an approximation to $\boldsymbol{u}\left(t_{i+1}\right)$, and $p^{i+1}$, an approximation to $p\left(t_{i+1}\right)$, by solving the following time-discrete version of equations $(2.1)-(2.3)$.

(3.1) $\alpha \frac{\boldsymbol{u}^{i+1}-\boldsymbol{u}^{i}}{\Delta t_{i+1}}+\beta \boldsymbol{u}^{i+1}+\nabla p^{i+1}=0, \quad$ in $\Omega^{i}, \quad \operatorname{div} \boldsymbol{u}^{i+1}=0, \quad$ in $\Omega^{i}, \quad p^{i+1}=\kappa^{i+1}+E^{i}, \quad$ on $\Gamma^{i}$.

Note that the updated velocity $\boldsymbol{u}^{i+1}$ will be defined on the current domain $\Omega^{i}$. Thus, since $\boldsymbol{u}^{i}$ has been determined on $\Omega^{i-1}$, it must first be extended to $\Omega^{i}$ in order to be used in (3.1). This will be discussed further below. In this equation, $\kappa^{i+1}$, which represents an approximation to the curvature $\kappa\left(\Gamma^{i+1}\right)$ of $\Gamma^{i+1}$, is also an unknown in the problem. Additional equations must be specified to determine it. Another unknown in our problem is $\boldsymbol{X}^{i+1}(\cdot)$, a parametrization of the next approximate interface $\Gamma^{i+1}$ at time $t_{i+1}$. To develop equations determining $\boldsymbol{X}^{i+1}(\cdot)$, we first approximate $(2.4)$ by the difference equation

$$
\boldsymbol{X}^{i+1}(s)=\boldsymbol{X}^{i}(s)+\Delta t_{i+1}\left[\boldsymbol{u}^{i+1}\left(\boldsymbol{X}^{i}(s)\right) \cdot \boldsymbol{n}^{i}\left(\boldsymbol{X}^{i}(s)\right)\right] \boldsymbol{n}^{i}\left(\boldsymbol{X}^{i}(s)\right) .
$$

Once $\boldsymbol{X}^{i+1}$ is determined, we define the interface at time $t_{i+1}$ by

$$
\Gamma^{i+1}=\left\{\boldsymbol{X}^{i+1}(s): s \in I\right\}
$$


and the domain $\Omega^{i+1}$ at time $t_{i+1}$ to be the domain enclosed by $\Gamma^{i+1}$. Writing $\boldsymbol{x}=\boldsymbol{X}^{i}(s) \in \Gamma^{i}$, we can also write $(3.2)$ in the form

$$
\boldsymbol{X}^{i+1} \circ\left(\boldsymbol{X}^{i}\right)^{-1}(\boldsymbol{x})=\boldsymbol{X}^{i} \circ\left(\boldsymbol{X}^{i}\right)^{-1}(\boldsymbol{x})+\Delta t_{i+1}\left[\boldsymbol{u}^{i+1}(\boldsymbol{x}) \cdot \boldsymbol{n}^{i}(\boldsymbol{x})\right] \boldsymbol{n}^{i}(\boldsymbol{x}) .
$$

Note that we can write $\boldsymbol{x}$ as the identity map on $\Gamma$, namely $\operatorname{id}_{\Gamma}$.

3.2. Curvature. To obtain additional equations to determine $\kappa^{i+1}$, we recall that the vector curvature of $\Gamma^{i}$ and $\Gamma^{i+1}$ are defined by

$$
\kappa\left(\Gamma^{i+1}\right) \boldsymbol{n}^{i+1}=-\Delta_{\Gamma^{i+1}}\left(\boldsymbol{X}^{i+1} \circ\left(\boldsymbol{X}^{i+1}\right)^{-1}\right), \quad \kappa\left(\Gamma^{i}\right) \boldsymbol{n}^{i}=-\Delta_{\Gamma^{i}}\left(\boldsymbol{X}^{i} \circ\left(\boldsymbol{X}^{i}\right)^{-1}\right) .
$$

Using the first of these equations (a fully implicit approach) is problematic, since the domain $\Omega^{i+1}$ is not known a priori. We follow [2] and use the semi-implicit discretization

$$
\begin{gathered}
\kappa^{i+1} \boldsymbol{n}^{i}=-\Delta_{\Gamma^{i}}\left(\boldsymbol{X}^{i+1} \circ\left(\boldsymbol{X}^{i}\right)^{-1}\right), \\
\kappa^{i+1}=-\Delta_{\Gamma^{i}}\left(\boldsymbol{X}^{i+1} \circ\left(\boldsymbol{X}^{i}\right)^{-1}\right) \cdot \boldsymbol{n}^{i} .
\end{gathered}
$$

Because of this approximation, $\kappa^{i+1}$ will only be an approximation of $\kappa\left(\Gamma^{i+1}\right)$.

3.3. Mapping the Domain. The model (3.1) is posed on a time-dependent domain, so a map is needed to go from $\Omega^{i}$ to $\Omega^{i+1}$. This is partially specified by (3.4) for mapping the boundary $\Gamma^{i}$ to $\Gamma^{i+1}$. Mapping the interior of $\Omega^{i}$ is done in the following way.

Let $\overline{\Omega^{i}}$ be compactly embedded in a fixed "hold-all" domain $\Omega_{\mathrm{H}}$ and let $\boldsymbol{D}^{i+1}: \Omega_{\mathrm{H}} \rightarrow \mathbb{R}^{2}$ be a displacement function such that

$$
\begin{aligned}
\boldsymbol{D}^{i+1} & =\boldsymbol{X}^{i+1}-\boldsymbol{X}^{i}, \text { on } \Gamma^{i} \equiv \partial \Omega^{i}, \\
& =\mathbf{0}, \text { on } \partial \Omega_{\mathrm{H}}, \\
& =\text { harmonic extension, in } \Omega_{\mathrm{H}},
\end{aligned}
$$

i.e., we take a smooth extension of the boundary data $\boldsymbol{X}^{i+1}-\boldsymbol{X}^{i}$ into the interior of $\Omega^{i}$ and the exterior $\Omega_{\mathrm{H}} \backslash \Omega^{i}$. There are many ways to extend a function; a harmonic extension is a convenient choice because of its smoothness. In the fully discrete scheme, $\boldsymbol{D}^{i+1}$ will act as a mesh displacement function for updating the discrete domain (mesh).

The domain $\Omega^{i+1}$ is defined in the following way. Assuming we have solved (3.1) on $\Omega^{i},(3.4)$ on $\Gamma^{i}$, and (3.7) on $\Omega^{i}$, we define

$$
\Omega^{i+1}:=\left\{\boldsymbol{x} \in \mathbb{R}^{2}: \boldsymbol{x}=\boldsymbol{x}^{i}+\boldsymbol{D}^{i+1}\left(\boldsymbol{x}^{i}\right), \text { for some } \boldsymbol{x}^{i} \in \Omega^{i}\right\},
$$

which is equivalent to writing

$$
\Omega^{i+1}:=\boldsymbol{Q}^{i+1}\left(\Omega^{i}\right), \quad \boldsymbol{Q}^{i+1}:=\left(\operatorname{id}_{\Omega_{\mathrm{H}}}+\boldsymbol{D}^{i+1}\right) .
$$

$\boldsymbol{Q}^{i+1}$ is a perturbation of the identity and is a continuous bijective map from $\Omega_{\mathrm{H}}$ to $\Omega_{\mathrm{H}}$, provided $\left\|\boldsymbol{D}^{i+1}\right\|_{L^{\infty}}$ is sufficiently small $[13,36,37,26]$. This is easily satisfied if $\Delta t_{i+1}$ is sufficiently small, because $\boldsymbol{D}^{i+1}=$ $O\left(\Delta t_{i+1}\right)$ and the harmonic extension obeys the maximum principle. In particular, if $\Omega^{i}$ is simply connected, then so is $\Omega^{i+1}$ (because $\boldsymbol{Q}^{i+1}$ is a continuous bijective map).

With the above considerations, we can now describe the mapping of $\boldsymbol{u}^{i+1}$ from $\Omega^{i}$ to $\Omega^{i+1}$ (recall that $\boldsymbol{u}^{i+1}$ is computed on $\Omega^{i}$ ). Let $\hat{\boldsymbol{u}}^{i+1}: \Omega^{i+1} \rightarrow \mathbb{R}^{2}$ be defined by

$$
\hat{\boldsymbol{u}}^{i+1}(\boldsymbol{x}):=\boldsymbol{u}^{i+1} \circ\left(\boldsymbol{Q}^{i+1}\right)^{-1}(\boldsymbol{x}), \quad \text { for all } \boldsymbol{x} \in \Omega^{i+1} .
$$

In other words, we treat the time derivative $d / d t$ in $(3.1)$ in a Lagrangian frame $[36,37]$, which can be thought of as an Arbitrary-Lagrangian-Eulerian (ALE) method [35]. [35]. 
Remarks. One typical aspect of ALE methods is to include an additional convective term in (3.1) to account for the fact that the domain $\Omega$ does not deform based on the velocity $\boldsymbol{u}$ (recall that we use the harmonic extension in (3.7) to define the new domain). The reason we do not include an ALE correction comes from a modeling consideration. The model (3.1) is obtained from the full 3-D Navier-Stokes equations via averaging along the channel height of the device and a suitable non-dimensionalization [42]. When nondimensionalizing the equations, one finds that a small parameter $\alpha$ multiplies the material derivative term of the (averaged) 2-D Navier-Stokes equations (relative to the $\beta$ parameter). $\alpha$ has the same value regardless of the presence of an ALE correction term. Hence, we may drop the convective term (and ALE correction) as part of deriving the model.

This also implies that we should drop the pure time-derivative term $\partial_{t} \boldsymbol{u}$. However, even though it is now non-dimensional, $\partial_{t} \boldsymbol{u}$ may have a large magnitude if there is a fast time-scale in the problem, i.e., $O\left(\partial_{t} \boldsymbol{u}\right) \gg 1$ if the relevant time-scale is much smaller than the natural time scale given by the length scale divided by the average droplet velocity scale. This may be caused by high frequency boundary actuation, i.e., the boundary function E oscillates rapidly or changes suddenly in time.

\section{VARIATIONAL FORMULATION AND WELL-POSEDNESS OF THE DISCRETE TIME PROBLEM}

4.1. Weak Formulation. Let $(\cdot, \cdot)_{\Omega}$ denote the $L^{2}$ inner product on $\Omega$ and $\langle\cdot, \cdot\rangle_{\Gamma}$ the duality pairing between $H^{-1 / 2}(\Gamma)$ and $H^{1 / 2}(\Gamma)$ or the $L^{2}(\Gamma)$ inner product, if both functions belong to $L^{2}(\Gamma)$. If $\kappa^{i+1} \in H^{1 / 2}\left(\Gamma^{i}\right)$ were known, a variational formulation of (3.1) is given by:

Find $\boldsymbol{u}^{i+1} \in H\left(\operatorname{div}, \Omega^{i}\right), p \in L^{2}\left(\Omega^{i}\right)$ such that

$$
\begin{aligned}
\left(\frac{\alpha}{\Delta t_{i+1}}+\beta\right)\left(\boldsymbol{u}^{i+1}, \boldsymbol{v}\right)_{\Omega^{i}} & -\left(p^{i+1}, \operatorname{div} \boldsymbol{v}\right)_{\Omega^{i}}+\left\langle\boldsymbol{v} \cdot \boldsymbol{n}^{i}, \kappa^{i+1}\right\rangle_{\Gamma^{i}} \\
& =\frac{\alpha}{\Delta t_{i+1}}\left(\boldsymbol{u}^{i}, \boldsymbol{v}\right)_{\Omega^{i}}-\left\langle\boldsymbol{v} \cdot \boldsymbol{n}^{i}, E^{i}\right\rangle_{\Gamma^{i}}, \quad \boldsymbol{v} \in H\left(\operatorname{div}, \Omega^{i}\right), \\
\left(\operatorname{div} \boldsymbol{u}^{i+1}, q\right)_{\Omega^{i}} & =0, \quad q \in L^{2}\left(\Omega^{i}\right) .
\end{aligned}
$$

From (3.4), we get for $\mu \in H^{1 / 2}\left(\Gamma^{i}\right)$,

$$
\frac{1}{\Delta t_{i+1}}\left\langle\boldsymbol{X}^{i+1} \circ\left(\boldsymbol{X}^{i}\right)^{-1}(\boldsymbol{x}) \cdot \boldsymbol{n}^{i}, \mu\right\rangle_{\Gamma^{i}}-\left\langle\boldsymbol{u}^{i+1}(\boldsymbol{x}) \cdot \boldsymbol{n}^{i}(\boldsymbol{x}), \mu\right\rangle_{\Gamma^{i}}=\frac{1}{\Delta t_{i+1}}\left\langle\boldsymbol{X}^{i} \circ\left(\boldsymbol{X}^{i}\right)^{-1}(\boldsymbol{x}) \cdot \boldsymbol{n}^{i}, \mu\right\rangle_{\Gamma^{i}}
$$

Using the semi-implicit discretization (3.5) for $\kappa^{i+1} \boldsymbol{n}^{i}$, and integrating by parts, we get

$$
\left.\left\langle\nabla_{\Gamma^{i}}\left[\boldsymbol{X}^{i+1} \circ\left(\boldsymbol{X}^{i}\right)^{-1}\right)\right], \nabla_{\Gamma^{i}} \boldsymbol{Y}\right\rangle_{\Gamma^{i}}-\left\langle\kappa^{i+1} \boldsymbol{n}^{i}, \boldsymbol{Y}\right\rangle_{\Gamma^{i}}=0, \quad \boldsymbol{Y} \in \boldsymbol{H}^{1}\left(\Gamma^{i}\right) .
$$

Remarks. The following sections analyze the semi-discrete and fully discrete EWOD problems during a single time step of the overall time-dependent method. Therefore, to simplify notation, we will drop the time-index " $i$ " notation and treat $\Omega$ and $\Gamma$ as given domains.

Moreover, $\kappa^{i+1}$ plays the role of a Lagrange multiplier on $\Gamma$ to enforce the constraint in (4.1). It is not the true curvature, though one would hope that it is a useful approximation of the curvature. So we will replace $\kappa^{i+1}$ by $\lambda$ to emphasize this fact.

In addition, note that $\boldsymbol{X}^{i+1} \circ\left(\boldsymbol{X}^{i}\right)^{-1}$ is a solution variable of the weak formulation. A separate step (definition) must be made in the time-dependent method that says $\boldsymbol{X}^{i+1}$ parameterizes the boundary of the domain at the next time-step (see additional remarks below). Thus, we will replace $\boldsymbol{X}^{i+1} \circ\left(\boldsymbol{X}^{i}\right)^{-1}$ by $\boldsymbol{W}$ in the weak formulation below to emphasize this fact. Furthermore, $\boldsymbol{X}^{i} \circ\left(\boldsymbol{X}^{i}\right)^{-1}$ is nothing more than the identity map on $\Gamma: \mathrm{id}_{\Gamma}$. 
Setting $\Delta t_{i+1} \equiv \Delta t$, and making the following substitutions

$$
\boldsymbol{u}^{i+1} \equiv \boldsymbol{u}, \quad \boldsymbol{u}^{i} \equiv \boldsymbol{U}, \quad p^{i+1} \equiv p, \quad \text { in } \Omega, \quad \kappa^{i+1} \equiv \lambda, \quad \boldsymbol{X}^{i+1} \circ\left(\boldsymbol{X}^{i}\right)^{-1} \equiv \boldsymbol{W}, \quad \text { on } \Gamma,
$$

we then have the following new variational (weak) formulation:

Find $\boldsymbol{u} \in H(\operatorname{div}, \Omega), p \in L^{2}(\Omega), \lambda \in H^{1 / 2}(\Gamma), \boldsymbol{W} \in \boldsymbol{H}^{1}(\Gamma)$, such that

$$
\begin{gathered}
\left(\frac{\alpha}{\Delta t}+\beta\right)(\boldsymbol{u}, \boldsymbol{v})_{\Omega}-(p, \operatorname{div} \boldsymbol{v})_{\Omega}+\langle\boldsymbol{v} \cdot \boldsymbol{n}, \lambda\rangle_{\Gamma}=\frac{\alpha}{\Delta t}(\boldsymbol{U}, \boldsymbol{v})_{\Omega}-\langle\boldsymbol{v} \cdot \boldsymbol{n}, E\rangle_{\Gamma}, \quad \boldsymbol{v} \in H(\operatorname{div}, \Omega), \\
(\operatorname{div} \boldsymbol{u}, q)_{\Omega}=0, \quad q \in L^{2}(\Omega) . \\
\frac{1}{\Delta t}\langle\boldsymbol{W} \cdot \boldsymbol{n}, \mu\rangle_{\Gamma}-\langle\boldsymbol{u} \cdot \boldsymbol{n}, \mu\rangle_{\Gamma}=\frac{1}{\Delta t}\left\langle\operatorname{id}_{\Gamma} \cdot \boldsymbol{n}, \mu\right\rangle_{\Gamma}, \quad \mu \in H^{1 / 2}(\Gamma), \\
\frac{1}{\Delta t}\left\langle\nabla_{\Gamma} \boldsymbol{W}, \nabla_{\Gamma} \boldsymbol{Y}\right\rangle_{\Gamma}-\frac{1}{\Delta t}\langle\lambda, \boldsymbol{n} \cdot \boldsymbol{Y}\rangle_{\Gamma}=0, \quad \boldsymbol{Y} \in \boldsymbol{H}^{1}(\Gamma) .
\end{gathered}
$$

Remarks. In this context, the time-dependent (discrete in time) formulation is as follows.

(1) Given $\Omega^{i}$ and $\Gamma^{i}$, we solve equations (4.2)-(4.5) on $\Omega^{i}$ and $\Gamma^{i}$ and obtain the function $\boldsymbol{W}: \Gamma^{i} \rightarrow \mathbb{R}^{2}$.

(2) Set $\boldsymbol{X}^{i+1}:=\boldsymbol{W}$.

(3) Define $\Gamma^{i+1}$ by (3.3) using $\boldsymbol{X}^{i+1}$.

(4) Define $\Omega^{i+1}$ as the interior of $\Gamma^{i+1}$.

(5) Go back to step 1 and repeat.

This generates a sequence of domains that approximates the time-dependent flow described in Section 3.

\subsection{Abstract Formulation. Defining}

$$
\begin{gathered}
\boldsymbol{\Sigma}=H(\operatorname{div}, \Omega) \times \boldsymbol{H}^{1}(\Gamma), \quad \Theta=L^{2}(\Omega) \times H^{1 / 2}(\Gamma), \\
a(\boldsymbol{u}, \boldsymbol{W} ; \boldsymbol{v}, \boldsymbol{Y})=\left(\frac{\alpha}{\Delta t}+\beta\right)(\boldsymbol{u}, \boldsymbol{v})_{\Omega}+\frac{1}{\Delta t}\left\langle\nabla_{\Gamma} \boldsymbol{W}, \nabla_{\Gamma} \boldsymbol{Y}\right\rangle_{\Gamma}, \\
b_{1}(p, \lambda ; \boldsymbol{v})=-(p, \operatorname{div} \boldsymbol{v})_{\Omega}+\langle\boldsymbol{v} \cdot \boldsymbol{n}, \lambda\rangle_{\Gamma}, \quad b_{2}(\lambda ; \boldsymbol{Y})=-\frac{1}{\Delta t}\langle\lambda, \boldsymbol{n} \cdot \boldsymbol{Y}\rangle_{\Gamma}, \\
b(p, \lambda ; \boldsymbol{v}, \boldsymbol{Y})=b_{1}(p, \lambda ; \boldsymbol{v})+b_{2}(\lambda ; \boldsymbol{Y}),
\end{gathered}
$$

and

$$
F(\boldsymbol{v}, \boldsymbol{Y})=\frac{\alpha}{\Delta t}(\boldsymbol{U}, \boldsymbol{v})_{\Omega}-\langle\boldsymbol{v} \cdot \boldsymbol{n}, E\rangle_{\Gamma}, \quad G(q, \mu)=-\frac{1}{\Delta t}\left\langle\operatorname{id}_{\Gamma} \cdot \boldsymbol{n}, \mu\right\rangle_{\Gamma},
$$

the variational formulation of equations (4.2)-(4.5) has the following saddle-point structure.

Find $(\boldsymbol{u}, \boldsymbol{W}) \in \boldsymbol{\Sigma}$ and $(p, \lambda) \in \Theta$, such that

$$
\begin{aligned}
a(\boldsymbol{u}, \boldsymbol{W} ; \boldsymbol{v}, \boldsymbol{Y})+b(p, \lambda ; \boldsymbol{v}, \boldsymbol{Y}) & =F(\boldsymbol{v}, \boldsymbol{Y}), \quad(\boldsymbol{v}, \boldsymbol{Y}) \in \boldsymbol{\Sigma}, \\
b(q, \mu ; \boldsymbol{u}, \boldsymbol{W}) & =G(q, \mu), \quad(q, \mu) \in \Theta .
\end{aligned}
$$

4.3. Norms. To show well-posedness of the semi-discrete problem (4.6)-(4.7), we check the standard conditions for saddle-point problems of this form (c.f. [8]). To do so, we first define the following norm on $\boldsymbol{H}^{1}(\Gamma)$. We set

$$
|\boldsymbol{W}|_{H_{*}^{1}(\Gamma)}=\left(\left|\nabla_{\Gamma} \boldsymbol{W}\right|_{L^{2}(\Gamma)}^{2}+\left|\langle\boldsymbol{W} \cdot \boldsymbol{n}, x\rangle_{\Gamma}\right|^{2}+\left|\langle\boldsymbol{W} \cdot \boldsymbol{n}, y\rangle_{\Gamma}\right|^{2}\right)^{1 / 2}
$$

The following proposition shows that this is indeed equivalent to the standard norm on $\boldsymbol{H}^{1}(\Gamma)$.

Proposition 4.1. There exists a constant $\zeta>0$ such that

$$
\frac{1}{\zeta}|\boldsymbol{W}|_{H^{1}(\Gamma)} \leq|\boldsymbol{W}|_{H_{*}^{1}(\Gamma)} \leq \zeta|\boldsymbol{W}|_{H^{1}(\Gamma)},
$$

where $|\boldsymbol{W}|_{H^{1}(\Gamma)}^{2}=\left|\nabla_{\Gamma} \boldsymbol{W}\right|_{L^{2}(\Gamma)}^{2}+|\boldsymbol{W}|_{L^{2}(\Gamma)}^{2}$. 
Proof. It is straightforward to show by Cauchy-Schwarz that

$$
\left|\langle\boldsymbol{W} \cdot \boldsymbol{n}, x\rangle_{\Gamma}\right|^{2}+\left|\langle\boldsymbol{W} \cdot \boldsymbol{n}, y\rangle_{\Gamma}\right|^{2} \leq\left(\int_{\Gamma}\left(x^{2}+y^{2}\right)\right)|\boldsymbol{W}|_{L^{2}(\Gamma)}^{2}
$$

so we obtain the right inequality of (4.8).

Next, let $\boldsymbol{W}^{A}=\frac{1}{|\Gamma|} \int_{\Gamma} \boldsymbol{W}$. Using the arithmetic-geometric mean inequality, we have for $\mu=x$ and $\mu=y$, and any $0 \leq \delta<1$,

$$
2\left|\left\langle\left[\boldsymbol{W}-\boldsymbol{W}^{A}\right] \cdot \boldsymbol{n}, \mu\right\rangle\right|\left|\left\langle\boldsymbol{W}^{A} \cdot \boldsymbol{n}, \mu\right\rangle\right| \leq \frac{1}{1-\delta}\left|\left\langle\left[\boldsymbol{W}-\boldsymbol{W}^{A}\right] \cdot \boldsymbol{n}, \mu\right\rangle\right|^{2}+(1-\delta)\left|\left\langle\boldsymbol{W}^{A} \cdot \boldsymbol{n}, \mu\right\rangle\right|^{2} .
$$

Then,

$$
\begin{aligned}
|\boldsymbol{W}|_{H_{*}^{1}(\Gamma)}^{2}= & \left|\nabla_{\Gamma} \boldsymbol{W}\right|_{L^{2}(\Gamma)}^{2}+\left|\left\langle\left[\boldsymbol{W}-\boldsymbol{W}^{A}\right] \cdot \boldsymbol{n}, x\right\rangle\right|^{2}+\left|\left\langle\left[\boldsymbol{W}-\boldsymbol{W}^{A}\right] \cdot \boldsymbol{n}, y\right\rangle\right|^{2}+\left|\left\langle\boldsymbol{W}^{A} \cdot \boldsymbol{n}, x\right\rangle\right|^{2}+\left|\left\langle\boldsymbol{W}^{A} \cdot \boldsymbol{n}, y\right\rangle\right|^{2} \\
& +2\left|\left\langle\left[\boldsymbol{W}-\boldsymbol{W}^{A}\right] \cdot \boldsymbol{n}, x\right\rangle\right|\left|\left\langle\boldsymbol{W}^{A} \cdot \boldsymbol{n}, x\right\rangle\right|+2\left|\left\langle\left[\boldsymbol{W}-\boldsymbol{W}^{A}\right] \cdot \boldsymbol{n}, y\right\rangle\right|\left|\left\langle\boldsymbol{W}^{A} \cdot \boldsymbol{n}, y\right\rangle\right| \\
\geq & \left|\nabla_{\Gamma} \boldsymbol{W}\right|_{L^{2}(\Gamma)}^{2}-\frac{\delta}{1-\delta}\left[\left|\left\langle\left[\boldsymbol{W}-\boldsymbol{W}^{A}\right] \cdot \boldsymbol{n}, x\right\rangle\right|^{2}+\left[\left|\left\langle\left[\boldsymbol{W}-\boldsymbol{W}^{A}\right] \cdot \boldsymbol{n}, y\right\rangle\right|\right]^{2}\right. \\
& \quad+\delta\left[\left|\left\langle\boldsymbol{W}^{A} \cdot \boldsymbol{n}, x\right\rangle\right|^{2}+\left|\left\langle\boldsymbol{W}^{A} \cdot \boldsymbol{n}, y\right\rangle\right|^{2}\right] \\
\geq & {\left[1-C \frac{\delta}{1-\delta}\right]\left|\nabla_{\Gamma} \boldsymbol{W}\right|_{L^{2}(\Gamma)}^{2}+\delta\left|\boldsymbol{W}^{A}\right|^{2}|\Omega|^{2} }
\end{aligned}
$$

where we used Cauchy-Schwarz and a standard Poincaré inequality [15]. Choosing $\delta$ sufficiently small and applying Poincaré's inequality again leads to

$$
|\boldsymbol{W}|_{H_{*}^{1}(\Gamma)}^{2} \geq \widetilde{C}\left(\left|\boldsymbol{W}-\boldsymbol{W}^{A}\right|_{L^{2}(\Gamma)}^{2}+\left|\boldsymbol{W}^{A}\right|_{L^{2}(\Gamma)}^{2}\right)=\widetilde{C}|\boldsymbol{W}|_{L^{2}(\Gamma)}^{2}
$$

The left inequality of (4.8) then follows by

$$
|\boldsymbol{W}|_{H^{1}(\Gamma)}^{2}=\left|\nabla_{\Gamma} \boldsymbol{W}\right|_{L^{2}(\Gamma)}^{2}+|\boldsymbol{W}|_{L^{2}(\Gamma)}^{2} \leq\left|\nabla_{\Gamma} \boldsymbol{W}\right|_{L^{2}(\Gamma)}^{2}+\frac{1}{\widetilde{C}}|\boldsymbol{W}|_{H_{*}^{1}(\Gamma)}^{2} \leq\left(1+\frac{1}{\widetilde{C}}\right)|\boldsymbol{W}|_{H_{*}^{1}(\Gamma)}^{2} .
$$

We then define

$$
\|(\boldsymbol{u}, \boldsymbol{W})\|_{\boldsymbol{\Sigma}}^{2}=\left(\frac{\alpha}{\Delta t}+\beta\right)\|\boldsymbol{u}\|_{H(\operatorname{div}, \Omega)}^{2}+\frac{1}{\Delta t}|\boldsymbol{W}|_{H_{*}^{1}(\Gamma)}^{2} .
$$

Next, we define the norm

$$
|\lambda|_{*, \Gamma}=\sup _{\boldsymbol{Z} \in H^{1}(\Gamma)} \frac{\langle\lambda \boldsymbol{n}, \boldsymbol{Z}\rangle_{\Gamma}}{|\boldsymbol{Z}|_{H_{*}^{1}(\Gamma)}}
$$

Note that $|\lambda|_{*, \Gamma}=\left|\boldsymbol{Y}_{0}\right|_{H_{*}^{1}(\Gamma)}$, where $\boldsymbol{Y}_{0} \in H^{1}(\Gamma)$ is the solution of

$$
\left\langle\nabla_{\Gamma} \boldsymbol{Y}_{0}, \nabla_{\Gamma} \boldsymbol{Z}\right\rangle_{\Gamma}+\left\langle\boldsymbol{Y}_{0} \cdot \boldsymbol{n}, x\right\rangle_{\Gamma}\langle\boldsymbol{Z} \cdot \boldsymbol{n}, x\rangle_{\Gamma}+\left\langle\boldsymbol{Y}_{0} \cdot \boldsymbol{n}, y\right\rangle_{\Gamma}\langle\boldsymbol{Z} \cdot \boldsymbol{n}, y\rangle_{\Gamma}=\langle\lambda \boldsymbol{n}, \boldsymbol{Z}\rangle_{\Gamma}, \quad \boldsymbol{Z} \in H^{1}(\Gamma)
$$

Finally, we define

$$
\|(p, \lambda)\|_{\Theta}^{2}=\|p-\hat{p}\|_{L^{2}(\Omega)}^{2}+|\lambda-\hat{\lambda}|_{H^{1 / 2}(\Gamma)}^{2}+|\hat{p}-\hat{\lambda}|^{2}+(1 / \Delta t)|\lambda|_{*, \Gamma}^{2},
$$

where $\hat{p}=(1 /|\Omega|) \int_{\Omega} p d x$ and $\hat{\lambda}=(1 /|\Gamma|) \int_{\Gamma} \lambda d \Gamma$. It is easy to check that $\|(p, \lambda)\|_{\Theta}$ is in fact a norm on $\Theta$. 
4.4. Continuity. We next establish the boundedness of the bilinear forms $a$ and $b$ and the linear functionals $F$ and $G$.

Lemma 4.2.

$$
\begin{gathered}
|a(\boldsymbol{u}, \boldsymbol{W} ; \boldsymbol{v}, \boldsymbol{Y})| \leq\|(\boldsymbol{u}, \boldsymbol{W})\|_{\boldsymbol{\Sigma}}\|(\boldsymbol{v}, \boldsymbol{Y})\|_{\boldsymbol{\Sigma}}, \quad \forall(\boldsymbol{u}, \boldsymbol{W}),(\boldsymbol{v}, \boldsymbol{Y}) \in \boldsymbol{\Sigma}, \\
|b(p, \lambda ; \boldsymbol{v}, \boldsymbol{Y})| \leq C\|(p, \lambda)\|_{\Theta}\|(\boldsymbol{v}, \boldsymbol{Y})\|_{\boldsymbol{\Sigma}}, \quad \forall(p, \lambda) \in \Theta,(\boldsymbol{v}, \boldsymbol{Y}) \in \boldsymbol{\Sigma}, \\
|F(\boldsymbol{v}, \boldsymbol{Y})| \leq C\left(\sqrt{\frac{\alpha}{\Delta t}}\|\boldsymbol{U}\|_{L^{2}(\Omega)}+|E|_{H^{1 / 2}(\Gamma)}\right)\|(\boldsymbol{v}, \boldsymbol{Y})\|_{\boldsymbol{\Sigma}}, \quad \forall(\boldsymbol{v}, \boldsymbol{Y}) \in \boldsymbol{\Sigma}, \\
|G(q, \mu)| \leq \frac{1}{\Delta t}\left|\operatorname{id}_{\Gamma}\right|_{H_{*}^{1}(\Gamma)}|\mu|_{*, \Gamma}, \quad \forall(q, \mu) \in \Theta .
\end{gathered}
$$

Proof. The first result follows immediately from the Schwarz inequality. To obtain the second estimate, we write

$$
\begin{array}{r}
b_{1}(p, \lambda ; \boldsymbol{v})=-(p, \operatorname{div} \boldsymbol{v})_{\Omega}+\langle\boldsymbol{v} \cdot \boldsymbol{n}, \lambda\rangle_{\Gamma}=-(p-\hat{p}, \operatorname{div} \boldsymbol{v})_{\Omega}+\langle\boldsymbol{v} \cdot \boldsymbol{n}, \lambda-\hat{\lambda}\rangle_{\Gamma}+\langle\boldsymbol{v} \cdot \boldsymbol{n}, \hat{\lambda}-\hat{p}\rangle_{\Gamma} \\
\leq\|p-\hat{p}\|_{L^{2}(\Omega)}\|\boldsymbol{v}\|_{H(\operatorname{div}, \Omega)}+C\left[|\lambda-\hat{\lambda}|_{H^{1 / 2}(\Gamma)}+|\hat{\lambda}-\hat{p}|\right]|\boldsymbol{v} \cdot \boldsymbol{n}|_{H^{-1 / 2}(\Gamma)} \\
\leq C\left[\|p-\hat{p}\|_{L^{2}(\Omega)}+|\lambda-\hat{\lambda}|_{H^{1 / 2}(\Gamma)}+|\hat{\lambda}-\hat{p}|\right]\|\boldsymbol{v}\|_{H(\operatorname{div}, \Omega)}
\end{array}
$$

We also have

$$
b_{2}(\lambda ; \boldsymbol{Y}) \leq \frac{1}{\Delta t}|\lambda|_{*, \Gamma}|\boldsymbol{Y}|_{H_{*}^{1}(\Gamma)} .
$$

The bound on $b$ follows easily from these inequalities. Next, we have

$$
\begin{aligned}
& |F(\boldsymbol{v}, \boldsymbol{Y})| \leq \frac{\alpha}{\Delta t}\left|(\boldsymbol{U}, \boldsymbol{v})_{\Omega}\right|+\left|\langle\boldsymbol{v} \cdot \boldsymbol{n}, E\rangle_{\Gamma}\right| \leq \frac{\alpha}{\Delta t}\|\boldsymbol{U}\|_{L^{2}(\Omega)}\|\boldsymbol{v}\|_{L^{2}(\Omega)}+|E|_{H^{1 / 2}(\Gamma)}|\boldsymbol{v} \cdot \boldsymbol{n}|_{H^{-1 / 2}(\Gamma)} \\
& \leq \sqrt{\frac{\alpha}{\Delta t}}\|\boldsymbol{U}\|_{L^{2}(\Omega)} \sqrt{\frac{\alpha}{\Delta t}}\|\boldsymbol{v}\|_{L^{2}(\Omega)}+|E|_{H^{1 / 2}(\Gamma)}\|\boldsymbol{v}\|_{H(\operatorname{div}, \Omega)} \leq C\left(\sqrt{\frac{\alpha}{\Delta t}}\|\boldsymbol{U}\|_{L^{2}(\Omega)}+|E|_{H^{1 / 2}(\Gamma)}\right)\|(\boldsymbol{v}, \boldsymbol{Y})\| \boldsymbol{\Sigma} .
\end{aligned}
$$

Finally, note that (4.9) implies $\langle\mu \boldsymbol{n}, \boldsymbol{Z}\rangle_{\Gamma} \leq|\mu|_{*, \Gamma}|\boldsymbol{Z}|_{H_{*}^{1}(\Gamma)}$ which yields

$$
|G(q, \mu)|=\frac{1}{\Delta t}\left|\left\langle\operatorname{id}_{\Gamma} \cdot \boldsymbol{n}, \mu\right\rangle_{\Gamma}\right| \leq \frac{1}{\Delta t}|\mu|_{*, \Gamma}\left|\operatorname{id}_{\Gamma}\right|_{H_{*}^{1}(\Gamma)} .
$$

4.5. Existence and Uniqueness. To establish well-posedness of the semi-discrete problem (4.6)-(4.7), it is then enough by the theory presented in [8] to prove the following result.

Theorem 4.3. (i) Let $(\boldsymbol{u}, \boldsymbol{W}) \in \boldsymbol{\Sigma}$ with $b(q, \mu ; \boldsymbol{u}, \boldsymbol{W})=0$ for all $(q, \mu) \in \Theta$. Then

$$
a(\boldsymbol{u}, \boldsymbol{W} ; \boldsymbol{u}, \boldsymbol{W}) \geq c\|(\boldsymbol{u}, \boldsymbol{W})\|_{\boldsymbol{\Sigma}}^{2},
$$

(ii) For all $(p, \lambda) \in \Theta$,

$$
\sup _{(\boldsymbol{v}, \boldsymbol{Y}) \in \boldsymbol{\Sigma}} \frac{b(p, \lambda ; \boldsymbol{v}, \boldsymbol{Y})}{\|(\boldsymbol{v}, \boldsymbol{Y})\|_{\boldsymbol{\Sigma}}} \geq C\|(p, \lambda)\|_{\Theta}
$$

Proof. First note that

Let $\phi^{\mu}$ satisfy

$$
a(\boldsymbol{u}, \boldsymbol{W} ; \boldsymbol{u}, \boldsymbol{W})=\left(\frac{\alpha}{\Delta t}+\beta\right)\|\boldsymbol{u}\|_{L^{2}(\Omega)}^{2}+\frac{1}{\Delta t}\left|\nabla_{\Gamma} \boldsymbol{W}\right|_{L^{2}(\Gamma)}^{2}
$$

$$
\Delta \phi^{\mu}=0, \quad \text { in } \Omega, \quad \phi^{\mu}=\mu, \quad \text { on } \Gamma .
$$

Since $b(q, \mu, \boldsymbol{u}, \boldsymbol{W})=0$ for all $(q, \mu) \in \Theta, \operatorname{div} \boldsymbol{u}=0$ in $\Omega$, and for all $\mu \in H^{1 / 2}(\Gamma)$,

$$
(1 / \Delta t)\langle\boldsymbol{W} \cdot \boldsymbol{n}, \mu\rangle_{\Gamma}=\langle\boldsymbol{u} \cdot \boldsymbol{n}, \mu\rangle_{\Gamma}=\left(\boldsymbol{u}, \nabla \phi^{\mu}\right)_{\Omega} \leq\|\boldsymbol{u}\|_{L^{2}(\Omega)}\left\|\nabla \phi^{\mu}\right\|_{L^{2}(\Omega)} .
$$


Choosing $\mu=x$ and then $\mu=y$, and observing that $\phi^{x}=x$ and $\phi^{y}=y$, we get

$$
\frac{1}{\Delta t}\left[\left|\langle\boldsymbol{W} \cdot \boldsymbol{n}, x\rangle_{\Gamma}\right|^{2}+\left|\langle\boldsymbol{W} \cdot \boldsymbol{n}, y\rangle_{\Gamma}\right|^{2}\right]^{1 / 2} \leq\|\boldsymbol{u}\|_{L^{2}(\Omega)}\left[\left\|\nabla \phi^{x}\right\|_{L^{2}(\Omega)}^{2}+\left\|\nabla \phi^{y}\right\|_{L^{2}(\Omega)}^{2}\right]^{1 / 2}=\sqrt{2}|\Omega|^{1 / 2}\|\boldsymbol{u}\|_{L^{2}(\Omega)} .
$$

Combining these results, we get (assuming $\Delta t \leq 1$ )

$$
\begin{array}{r}
a(\boldsymbol{u}, \boldsymbol{W} ; \boldsymbol{u}, \boldsymbol{W}) \geq C\left[\left(\frac{\alpha}{\Delta t}+\beta\right)\|\boldsymbol{u}\|_{H(\operatorname{div}, \Omega)}^{2}+\frac{1}{\Delta t}\left|\nabla_{\Gamma} \boldsymbol{W}\right|_{L^{2}(\Gamma)}^{2}+\frac{1}{(\Delta t)^{2}}\left(\left|\langle\boldsymbol{W} \cdot \boldsymbol{n}, x\rangle_{\Gamma}\right|^{2}+\left|\langle\boldsymbol{W} \cdot \boldsymbol{n}, y\rangle_{\Gamma}\right|^{2}\right)\right] \\
\geq c\|(\boldsymbol{u}, \boldsymbol{W})\|_{\boldsymbol{\Sigma}}^{2} .
\end{array}
$$

To show that condition (ii) is satisfied, set $\boldsymbol{v}=\nabla \phi_{1}+\nabla \phi_{2}+\nabla \phi_{3}$, where $\phi_{1}$, $\phi_{2}$, and $\phi_{3}$ satisfy the boundary value problems

$$
\begin{gathered}
-\Delta \phi_{1}=p-\hat{p} \text { in } \Omega, \quad \frac{\partial \phi_{1}}{\partial n}=0 \text { on } \Gamma, \quad \int_{\Omega} \phi_{1} d x=0, \quad-\Delta \phi_{2}=0 \text { in } \Omega, \quad \phi_{2}=\lambda-\hat{\lambda} \text { on } \Gamma, \\
\Delta \phi_{3}=\frac{\hat{\lambda}-\hat{p}}{|\Omega|} \text { in } \Omega, \quad \frac{\partial \phi_{3}}{\partial n}=\frac{\hat{\lambda}-\hat{p}}{|\Gamma|} \text { on } \Gamma, \quad \int_{\Omega} \phi_{3} d x=0 .
\end{gathered}
$$

Then

Hence, we have

$$
-\operatorname{div} \boldsymbol{v}=p-\hat{p}-\frac{\hat{\lambda}-\hat{p}}{|\Omega|}, \quad \boldsymbol{v} \cdot \boldsymbol{n}=\frac{\hat{\lambda}-\hat{p}}{|\Gamma|}+\partial \phi_{2} / \partial \boldsymbol{n}
$$

$$
\begin{aligned}
-(p, \operatorname{div} \boldsymbol{v})_{\Omega}+\langle\boldsymbol{v} \cdot \boldsymbol{n}, \lambda\rangle_{\Gamma} & =(p, p-\hat{p})_{\Omega}-\frac{1}{|\Omega|}(p, \hat{\lambda}-\hat{p})_{\Omega}+\frac{1}{|\Gamma|}\langle\lambda, \hat{\lambda}-\hat{p}\rangle_{\Gamma}+\left\langle\partial \phi_{2} / \partial \boldsymbol{n}, \lambda\right\rangle_{\Gamma} \\
& =\|p-\hat{p}\|_{L^{2}(\Omega)}^{2}-\frac{1}{|\Omega|}(\hat{p}, \hat{\lambda}-\hat{p})_{\Omega}+\frac{1}{|\Gamma|}\langle\hat{\lambda}, \hat{\lambda}-\hat{p}\rangle_{\Gamma}+\left\langle\partial \phi_{2} / \partial \boldsymbol{n}, \lambda\right\rangle_{\Gamma} \\
& =\|p-\hat{p}\|_{L^{2}(\Omega)}^{2}+|\hat{\lambda}-\hat{p}|^{2}+\left\langle\partial \phi_{2} / \partial \boldsymbol{n}, \lambda-\hat{\lambda}\right\rangle_{\Gamma} \\
& =\|p-\hat{p}\|_{L^{2}(\Omega)}^{2}+|\hat{\lambda}-\hat{p}|^{2}+\left\langle\partial \phi_{2} / \partial \boldsymbol{n}, \phi_{2}\right\rangle_{\Gamma} \\
& =\|p-\hat{p}\|_{L^{2}(\Omega)}^{2}+|\hat{\lambda}-\hat{p}|^{2}+\left\|\nabla \phi_{2}\right\|_{L^{2}(\Omega)}^{2} .
\end{aligned}
$$

Since

$$
|\mu|_{H^{1 / 2}(\Gamma)}=\inf _{\substack{\psi \in H^{1}(\Omega) \\ \psi=\mu \text { on } \Gamma}}\|\psi\|_{H^{1}(\Omega)}, \quad \int_{\Gamma}(\lambda-\hat{\lambda}) d \Gamma=0
$$

we have

$$
|\lambda-\hat{\lambda}|_{H^{1 / 2}(\Gamma)} \leq\left\|\phi_{2}\right\|_{H^{1}(\Omega)} .
$$

By Green's formula, we have for all $\boldsymbol{w} \in H(\operatorname{div}, \Omega)$ that

$$
\left(\nabla \phi_{2}, \boldsymbol{w}\right)_{\Omega}+\left(\phi_{2}, \operatorname{div} \boldsymbol{w}\right)_{\Omega}=\langle\boldsymbol{w} \cdot \boldsymbol{n}, \lambda-\hat{\lambda}\rangle_{\Gamma} .
$$

Choosing $\boldsymbol{w}=\nabla W$, where $W$ satisfies

$$
\Delta W=\phi_{2} \text { in } \Omega, \quad \partial W / \partial n=|\Gamma|^{-1} \int_{\Omega} \phi_{2} \text { on } \Gamma, \quad \int_{\Omega} W=0,
$$

we have $\|\boldsymbol{w}\|_{H(\operatorname{div}, \Omega)} \leq \gamma\left\|\phi_{2}\right\|_{L^{2}(\Omega)}$, for some positive constant $\gamma$, and

$$
\left\|\phi_{2}\right\|_{L^{2}(\Omega)}^{2} \leq\left\|\nabla \phi_{2}\right\|_{L^{2}(\Omega)}\|\boldsymbol{w}\|_{L^{2}(\Omega)} \leq \gamma\left\|\nabla \phi_{2}\right\|_{L^{2}(\Omega)}\left\|\phi_{2}\right\|_{L^{2}(\Omega)} .
$$

Hence $\left\|\phi_{2}\right\|_{H^{1}(\Omega)} \leq \gamma\left\|\nabla \phi_{2}\right\|_{L^{2}(\Omega)}$, and so

$$
-(p, \operatorname{div} \boldsymbol{v})_{\Omega}+\langle\boldsymbol{v} \cdot \boldsymbol{n}, \lambda\rangle_{\Gamma} \geq\|p-\hat{p}\|_{L^{2}(\Omega)}^{2}+|\hat{\lambda}-\hat{p}|^{2}+\gamma^{-2}|\lambda-\hat{\lambda}|_{H^{1 / 2}(\Gamma)}^{2}
$$

and

$$
\begin{aligned}
\|\boldsymbol{v}\|_{H(\operatorname{div}, \Omega)} & \leq\left\|\nabla \phi_{1}\right\|_{L^{2}(\Omega)}+\left\|\Delta \phi_{1}\right\|_{L^{2}(\Omega)}+\left\|\nabla \phi_{2}\right\|_{L^{2}(\Omega)}+\left\|\nabla \phi_{3}\right\|_{L^{2}(\Omega)}+\left\|\Delta \phi_{3}\right\|_{L^{2}(\Omega)} \\
& \leq C\left(\|p-\hat{p}\|_{L^{2}(\Omega)}+|\hat{\lambda}-\hat{p}|+|\lambda-\hat{\lambda}|_{H^{1 / 2}(\Gamma)}\right) .
\end{aligned}
$$


Next, set $\boldsymbol{Y}=-\boldsymbol{Y}_{0}$, where $\boldsymbol{Y}_{0}$ is defined by (4.10). Then

$$
-\frac{1}{\Delta t}\langle\lambda \boldsymbol{n}, \boldsymbol{Y}\rangle_{\Gamma}=\frac{1}{\Delta t}\left|\boldsymbol{Y}_{0}\right|_{H_{*}^{1}, \Gamma}^{2}=\frac{1}{\Delta t}|\lambda|_{*, \Gamma}^{2} .
$$

Combining these results (recall the definition of $b$ ), we have

$$
\frac{b(p, \lambda ; \boldsymbol{v}, \boldsymbol{Y})}{\|(\boldsymbol{v}, \boldsymbol{Y})\|_{\boldsymbol{\Sigma}}} \geq C \frac{\|p-\hat{p}\|_{L^{2}(\Omega)}^{2}+\gamma^{-2}|\lambda-\hat{\lambda}|_{H^{1 / 2}(\Gamma)}^{2}+|\hat{\lambda}-\hat{p}|^{2}+(1 / \Delta t)|\lambda|_{*, \Gamma}^{2}}{\left[\left(\|p-\hat{p}\|_{L^{2}(\Omega)}^{2}+|\lambda-\hat{\lambda}|_{H^{1 / 2}(\Gamma)}^{2}+|\hat{\lambda}-\hat{p}|^{2}\right)+(1 / \Delta t)|\lambda|_{*, \Gamma}^{2}\right]^{1 / 2}} \geq C\|(p, \lambda)\|_{\Theta} .
$$

The assertion follows by taking the supremum.

\section{Description AND Well-Posedness of A FUlly discrete APPROXimation SCHEME}

We begin by approximating the domain $\Omega$ by a polygonal domain $\Omega_{h}$ with boundary $\Gamma_{h}$. A standard Galerkin approximation of equations (4.2)-(4.5) takes the form: Find $\boldsymbol{u}_{h} \in \boldsymbol{V}_{h} \subset H\left(\operatorname{div}, \Omega_{h}\right), p_{h} \in Q_{h} \subset$ $L^{2}\left(\Omega_{h}\right), \lambda_{h} \in \Lambda_{h} \subset H^{1 / 2}\left(\Gamma_{h}\right), \boldsymbol{W}_{h} \in \boldsymbol{\Upsilon}_{h} \subset \boldsymbol{H}^{1}\left(\Gamma_{h}\right)$ such that

$$
\begin{gathered}
\left(\frac{\alpha}{\Delta t}+\beta\right)\left(\boldsymbol{u}_{h}, \boldsymbol{v}\right)_{\Omega_{h}}-\left(p_{h}, \operatorname{div} \boldsymbol{v}\right)_{\Omega_{h}}+\left\langle\boldsymbol{v} \cdot \boldsymbol{n}, \lambda_{h}\right\rangle_{\Gamma_{h}}=\frac{\alpha}{\Delta t}\left(\boldsymbol{U}_{h}, \boldsymbol{v}\right)_{\Omega_{h}}-\langle\boldsymbol{v} \cdot \boldsymbol{n}, E\rangle_{\Gamma_{h}}, \quad \boldsymbol{v} \in \boldsymbol{V}_{h}, \\
\quad\left(\operatorname{div} \boldsymbol{u}_{h}, q\right)_{\Omega_{h}}=0, \quad q \in Q_{h} . \\
\frac{1}{\Delta t}\left\langle\boldsymbol{W}_{h} \cdot \boldsymbol{n}, \mu\right\rangle_{\Gamma_{h}}-\left\langle\boldsymbol{u}_{h} \cdot \boldsymbol{n}, \mu\right\rangle_{\Gamma_{h}}=\frac{1}{\Delta t}\left\langle\operatorname{id}_{\Gamma_{h}} \cdot \boldsymbol{n}, \mu\right\rangle_{\Gamma_{h}}, \quad \mu \in \Lambda_{h}, \\
\frac{1}{\Delta t}\left\langle\nabla_{\Gamma_{h}} \boldsymbol{W}_{h}, \nabla_{\Gamma_{h}} \boldsymbol{Y}\right\rangle_{\Gamma_{h}}-\frac{1}{\Delta t}\left\langle\lambda_{h}, \boldsymbol{n} \cdot \boldsymbol{Y}\right\rangle_{\Gamma_{h}}=0, \quad \boldsymbol{Y} \in \boldsymbol{\Upsilon}_{h} .
\end{gathered}
$$

Setting $\boldsymbol{\Sigma}_{h}=\boldsymbol{V}_{h} \times \boldsymbol{\Upsilon}_{h}$ and $\Theta_{h}=Q_{h} \times \Lambda_{h}$, and defining

$$
\begin{gathered}
a_{h}(\boldsymbol{u}, \boldsymbol{W} ; \boldsymbol{v}, \boldsymbol{Y})=\left(\frac{\alpha}{\Delta t}+\beta\right)(\boldsymbol{u}, \boldsymbol{v})_{\Omega_{h}}+\frac{1}{\Delta t}\left\langle\nabla_{\Gamma_{h}} \boldsymbol{W}, \nabla_{\Gamma_{h}} \boldsymbol{Y}\right\rangle_{\Gamma_{h}}, \\
b_{1 h}(p, \lambda ; \boldsymbol{v})=-(p, \operatorname{div} \boldsymbol{v})_{\Omega_{h}}+\langle\boldsymbol{v} \cdot \boldsymbol{n}, \lambda\rangle_{\Gamma_{h}}, \quad b_{2 h}(\lambda ; \boldsymbol{Y})=-\frac{1}{\Delta t}\langle\lambda, \boldsymbol{n} \cdot \boldsymbol{Y}\rangle_{\Gamma_{h}}, \\
b_{h}(p, \lambda ; \boldsymbol{v}, \boldsymbol{Y})=b_{1 h}(p, \lambda ; \boldsymbol{v})+b_{2 h}(\lambda ; \boldsymbol{Y}), \\
F_{h}(\boldsymbol{v}, \boldsymbol{Y})=\frac{\alpha}{\Delta t}\left(\boldsymbol{U}_{h}, \boldsymbol{v}\right)_{\Omega_{h}}-\langle\boldsymbol{v} \cdot \boldsymbol{n}, E\rangle_{\Gamma_{h}}, \quad G_{h}(q, \mu)=-\frac{1}{\Delta t}\left\langle\operatorname{id}_{\Gamma_{h}} \cdot \boldsymbol{n}, \mu\right\rangle_{\Gamma_{h}},
\end{gathered}
$$

we can rewrite the above in the form

Find $\left(\boldsymbol{u}_{h}, \boldsymbol{W}_{h}\right) \in \boldsymbol{\Sigma}_{h}$ and $\left(p_{h}, \lambda_{h}\right) \in \Theta_{h}$, such that

$$
\begin{aligned}
a_{h}\left(\boldsymbol{u}_{h}, \boldsymbol{W}_{h} ; \boldsymbol{v}, \boldsymbol{Y}\right)+b_{h}\left(p_{h}, \lambda_{h} ; \boldsymbol{v}, \boldsymbol{Y}\right) & =F_{h}(\boldsymbol{v}, \boldsymbol{Y}), \quad(\boldsymbol{v}, \boldsymbol{Y}) \in \boldsymbol{\Sigma}_{h}, \\
b_{h}\left(q, \mu ; \boldsymbol{u}_{h}, \boldsymbol{W}_{h}\right) & =G_{h}(q, \mu), \quad(q, \mu) \in \Theta_{h} .
\end{aligned}
$$

To establish well-posedness, we show that the discrete version of the conditions of Theorem 4.3 are satisfied. At this time, we make the following abstract assumptions about the choice of subspaces. Later in the paper, we consider specific choices of spaces that satisfy these assumptions. Let $\dot{\boldsymbol{V}}_{h}=\left\{\boldsymbol{v} \in \boldsymbol{V}_{h}: \boldsymbol{v} \cdot \boldsymbol{n}=0\right.$ on $\left.\Gamma_{h}\right\}$ and $\hat{Q}_{h}=\left\{q \in Q_{h}: \int_{\Omega_{h}} q d x=0\right\}$. We assume that $\operatorname{div} \boldsymbol{V}_{h}=Q_{h}$, $\operatorname{div} \stackrel{\circ}{\boldsymbol{V}}_{h}=\hat{Q}_{h}, \boldsymbol{V}_{h}$ contains continuous linear functions, and that $\left(\boldsymbol{V}_{h}, Q_{h}\right)$ satisfy the discrete inf-sup condition

$$
\sup _{v \in \boldsymbol{V}_{h}} \frac{(\operatorname{div} \boldsymbol{v}, p)_{\Omega_{h}}}{\|\boldsymbol{v}\|_{H\left(\operatorname{div}, \Omega_{h}\right)}} \geq c\|p\|_{L^{2}\left(\Omega_{h}\right)},
$$

with $c$ independent of $h$ and that an analogous condition is satisfied for the pair $\left(\dot{\boldsymbol{V}}_{h}, \hat{Q}_{h}\right)$. As for $\Lambda_{h}$, we assume it contains globally linear functions. 
We also require the following technical assumption, which is easily verified for standard choices of the spaces $\boldsymbol{V}_{h}$ and $Q_{h}$. For any $\phi_{h} \in Q_{h}$, there exists $\boldsymbol{w}_{h} \in \boldsymbol{V}_{h}$ satisfying

$$
\operatorname{div} \boldsymbol{w}_{h}=\phi_{h} \text { in } \Omega_{h}, \quad \boldsymbol{w}_{h} \cdot \boldsymbol{n}=\left|\Gamma_{h}\right|^{-1} \int_{\Omega_{h}} \phi_{h} \text { on } \Gamma_{h}, \quad\left\|\boldsymbol{w}_{h}\right\|_{H\left(\operatorname{div}, \Omega_{h}\right)} \leq C\left\|\phi_{h}\right\|_{L^{2}\left(\Omega_{h}\right)} .
$$

We will use a discrete analogue of $|\mu|_{H^{1 / 2}\left(\Gamma_{h}\right)}$ defined for $\mu \in \Lambda_{h}$ by

$$
|\mu|_{H_{h}^{1 / 2}\left(\Gamma_{h}\right)}=\inf _{(\boldsymbol{w}, \psi) \in Z_{h}(\mu)}\left(\|\boldsymbol{w}\|_{L^{2}\left(\Omega_{h}\right)}^{2}+\|\psi\|_{L^{2}\left(\Omega_{h}\right)}^{2}\right)^{1 / 2},
$$

where $Z_{h}(\mu)=\left\{(\boldsymbol{w}, \psi) \in \boldsymbol{V}_{h} \times Q_{h}\right\}$ satisfies

$$
(\boldsymbol{w}, \boldsymbol{v})_{\Omega_{h}}+(\psi, \operatorname{div} \boldsymbol{v})_{\Omega_{h}}=\langle\boldsymbol{v} \cdot \boldsymbol{n}, \mu\rangle_{\Gamma_{h}}, \quad \boldsymbol{v} \in \boldsymbol{V}_{h} .
$$

Note that if the infimum is achieved for $(\boldsymbol{w}, \psi)=\left(\boldsymbol{w}_{0}, \psi_{0}\right) \in Z_{h}(\mu)$, then for all $\mu \in \Lambda_{h}$ and $\boldsymbol{v} \in \boldsymbol{V}_{h}$,

$$
\begin{array}{r}
\langle\boldsymbol{v} \cdot \boldsymbol{n}, \mu\rangle_{\Gamma_{h}}=\left(\boldsymbol{w}_{0}, \boldsymbol{v}\right)_{\Omega_{h}}+\left(\psi_{0}, \operatorname{div} \boldsymbol{v}\right)_{\Omega_{h}} \leq\left(\left\|\boldsymbol{w}_{0}\right\|_{L^{2}\left(\Omega_{h}\right)}^{2}+\left\|\psi_{0}\right\|_{L^{2}\left(\Omega_{h}\right)}^{2}\right)^{1 / 2}\left(\|\boldsymbol{v}\|_{L^{2}\left(\Omega_{h}\right)}^{2}+\|\operatorname{div} \boldsymbol{v}\|_{L^{2}\left(\Omega_{h}\right)}^{2}\right)^{1 / 2} \\
\leq|\mu|_{H_{h}^{1 / 2}\left(\Gamma_{h}\right)}\|\boldsymbol{v}\|_{H\left(\operatorname{div}, \Omega_{h}\right)} .
\end{array}
$$

We also define a discrete analogue of $|\lambda|_{*, \Gamma}$ defined by

$$
|\lambda|_{*, h, \Gamma_{h}}=\sup _{\boldsymbol{Z} \in \boldsymbol{\Upsilon}_{h}} \frac{\langle\lambda \boldsymbol{n}, \boldsymbol{Z}\rangle_{\Gamma_{h}}}{|\boldsymbol{Z}|_{H_{*}^{1}\left(\Gamma_{h}\right)}} .
$$

Note, that $|\lambda|_{*, h, \Gamma}=\left|\boldsymbol{Y}_{0, h}\right|_{H_{*}^{1}\left(\Gamma_{h}\right)}$, where $\boldsymbol{Y}_{0, h} \in \boldsymbol{\Upsilon}_{h}$ is the solution of

(5.11) $\left\langle\nabla_{\Gamma_{h}} \boldsymbol{Y}_{0, h}, \nabla_{\Gamma_{h}} \boldsymbol{Z}\right\rangle_{\Gamma_{h}}+\left\langle\boldsymbol{Y}_{0, h} \cdot \boldsymbol{n}, x\right\rangle_{\Gamma_{h}}\langle\boldsymbol{Z} \cdot \boldsymbol{n}, x\rangle_{\Gamma_{h}}+\left\langle\boldsymbol{Y}_{0, h} \cdot \boldsymbol{n}, y\right\rangle_{\Gamma_{h}}\langle\boldsymbol{Z} \cdot \boldsymbol{n}, y\rangle_{\Gamma_{h}}=\langle\lambda \boldsymbol{n}, \boldsymbol{Z}\rangle_{\Gamma_{h}}, \quad \boldsymbol{Z} \in \boldsymbol{\Upsilon}_{h}$.

Finally, we define

$$
\begin{gathered}
\|(\boldsymbol{u}, \boldsymbol{W})\|_{\boldsymbol{\Sigma}_{h}}^{2}=\left(\frac{\alpha}{\Delta t}+\beta\right)\|\boldsymbol{u}\|_{H\left(\operatorname{div}, \Omega_{h}\right)}^{2}+\frac{1}{\Delta t}|\boldsymbol{W}|_{H_{*}^{1}\left(\Gamma_{h}\right)}^{2}, \\
\|(p, \lambda)\|_{\Theta_{h}}^{2}=\|p-\hat{p}\|_{L^{2}\left(\Omega_{h}\right)}^{2}+|\lambda-\hat{\lambda}|_{H_{h}^{1 / 2}\left(\Gamma_{h}\right)}^{2}+|\hat{p}-\hat{\lambda}|^{2}+(1 / \Delta t)|\lambda|_{*, h, \Gamma_{h}}^{2} .
\end{gathered}
$$

The following result, whose proof is analogous to the proof of Lemma 4.2, gives additional bounds for the bilinear form $b_{h}$ and the linear functionals $F_{h}$ and $G_{h}$.

Lemma 5.1.

$$
\begin{gathered}
\left|a_{h}(\boldsymbol{u}, \boldsymbol{W} ; \boldsymbol{v}, \boldsymbol{Y})\right| \leq\|(\boldsymbol{u}, \boldsymbol{W})\|_{\boldsymbol{\Sigma}_{h}}\|(\boldsymbol{v}, \boldsymbol{Y})\|_{\boldsymbol{\Sigma}_{h}}, \quad \forall(\boldsymbol{u}, \boldsymbol{W}),(\boldsymbol{v}, \boldsymbol{Y}) \in \boldsymbol{\Sigma}_{h}, \\
\left|b_{h}(p, \lambda ; \boldsymbol{v}, \boldsymbol{Y})\right| \leq C\|(p, \lambda)\|_{\Theta_{h}}\|(\boldsymbol{v}, \boldsymbol{Y})\|_{\boldsymbol{\Sigma}_{h}}, \quad \forall(p, \lambda) \in \Theta_{h}, \quad(\boldsymbol{v}, \boldsymbol{Y}) \in \boldsymbol{\Sigma}_{h}, \\
\left|F_{h}(\boldsymbol{v}, \boldsymbol{Y})\right| \leq C\left(\sqrt{\frac{\alpha}{\Delta t}}\left\|\boldsymbol{U}_{h}\right\|_{L^{2}(\Omega)}+|E|_{H^{1 / 2}\left(\Gamma_{h}\right)}\right)\|(\boldsymbol{v}, \boldsymbol{Y})\|_{\boldsymbol{\Sigma}_{h}}, \quad \forall(\boldsymbol{v}, \boldsymbol{Y}) \in \boldsymbol{\Sigma}_{h}, \\
\left|G_{h}(q, \mu)\right| \leq \frac{1}{\Delta t}\left|\operatorname{id}_{\Gamma_{h}}\right|_{H_{*}^{1}\left(\Gamma_{h}\right)}|\mu|_{*, h, \Gamma_{h}}, \quad \forall(q, \mu) \in \Theta_{h} .
\end{gathered}
$$

Theorem 5.2. (i) Let $\left(\boldsymbol{u}_{h}, \boldsymbol{W}_{h}\right) \in \boldsymbol{\Sigma}_{h}$ with $b_{h}\left(q, \mu ; \boldsymbol{u}_{h}, \boldsymbol{W}_{h}\right)=0$ for all $(q, \mu) \in \Theta_{h}$. Then there exists a constant $c$ independent of $h$, such that

$$
a_{h}\left(\boldsymbol{u}_{h}, \boldsymbol{W}_{h} ; \boldsymbol{u}_{h}, \boldsymbol{W}_{h}\right) \geq c\left\|\left(\boldsymbol{u}_{h}, \boldsymbol{W}_{h}\right)\right\|_{\boldsymbol{\Sigma}_{h}}^{2} .
$$

(ii) There exists a constant $C$ independent of $h$ such that

$$
\sup _{(\boldsymbol{v}, \boldsymbol{Y}) \in \boldsymbol{\Sigma}_{h}} \frac{b_{h}(p, \lambda ; \boldsymbol{v}, \boldsymbol{Y})}{\|(\boldsymbol{v}, \boldsymbol{Y})\|_{\boldsymbol{\Sigma}_{h}}} \geq C\|(p, \lambda)\|_{\Theta_{h}},
$$

for all $(p, \lambda) \in \Theta_{h}$. 
Proof. Let $\left(\boldsymbol{u}_{h}, \boldsymbol{W}_{h}\right) \in \boldsymbol{\Sigma}_{h}$ with $b\left(q, \mu, \boldsymbol{u}_{h}, \boldsymbol{W}_{h}\right)=0$ for all $(q, \mu) \in \Theta_{h}$. Now

$$
a\left(\boldsymbol{u}_{h}, \boldsymbol{W}_{h} ; \boldsymbol{u}_{h}, \boldsymbol{W}_{h}\right)=\left(\frac{\alpha}{\Delta t}+\beta\right)\left\|\boldsymbol{u}_{h}\right\|_{L^{2}\left(\Omega_{h}\right)}^{2}+\frac{1}{\Delta t}\left|\nabla_{\Gamma_{h}} \boldsymbol{W}_{h}\right|_{L^{2}\left(\Gamma_{h}\right)}^{2} .
$$

Since $b_{h}\left(q, \mu, \boldsymbol{u}_{h}, \boldsymbol{W}_{h}\right)=0$ for all $(q, \mu) \in \Theta_{h}$, and $\operatorname{div} \boldsymbol{V}_{h}=Q_{h}$, we have $\operatorname{div} \boldsymbol{u}_{h}=0$ in $\Omega_{h}$ and for all $\mu \in \Lambda_{h}$,

$$
(1 / \Delta t)\left\langle\boldsymbol{W}_{h} \cdot \boldsymbol{n}, \mu\right\rangle_{\Gamma_{h}}=\left\langle\boldsymbol{u}_{h} \cdot \boldsymbol{n}, \mu\right\rangle_{\Gamma_{h}} .
$$

However, since $x$ and $y \in \Lambda_{h}$, we may proceed exactly as in the continuous case to show that

$$
(1 / \Delta t)\left[\left|\left\langle\boldsymbol{W}_{h} \cdot \boldsymbol{n}, x\right\rangle_{\Gamma_{h}}\right|^{2}+\left|\left\langle\boldsymbol{W}_{h} \cdot \boldsymbol{n}, y\right\rangle_{\Gamma_{h}}\right|^{2}\right]^{1 / 2} \leq \sqrt{2}\left|\Omega_{h}\right|^{1 / 2}\left\|\boldsymbol{u}_{h}\right\|_{L^{2}\left(\Omega_{h}\right)} .
$$

Combining these results, we get (assuming $\Delta t \leq 1$ )

$$
\begin{aligned}
a\left(\boldsymbol{u}_{h}, \boldsymbol{W}_{h} ; \boldsymbol{u}_{h}, \boldsymbol{W}_{h}\right) \geq C\left(\left(\frac{\alpha}{\Delta t}+\beta\right)\right. & \left\|\boldsymbol{u}_{h}\right\|_{H\left(\operatorname{div}, \Omega_{h}\right)}^{2}+\frac{1}{\Delta t}\left|\nabla_{\Gamma_{h}} \boldsymbol{W}_{h}\right|_{L^{2}\left(\Gamma_{h}\right)}^{2} \\
& \left.+\frac{1}{\Delta t^{2}}\left(\left|\left\langle\boldsymbol{W}_{h} \cdot \boldsymbol{n}, x\right\rangle_{\Gamma_{h}}\right|^{2}+\left|\left\langle\boldsymbol{W}_{h} \cdot \boldsymbol{n}, y\right\rangle_{\Gamma_{h}}\right|^{2}\right)\right) \geq c\left\|\left(\boldsymbol{u}_{h}, \boldsymbol{W}_{h}\right)\right\|_{\boldsymbol{\Sigma}_{h}}^{2} .
\end{aligned}
$$

To show that condition (ii) is satisfied, set $\boldsymbol{v}_{h}=\boldsymbol{v}_{1, h}+\boldsymbol{v}_{2, h}+\boldsymbol{v}_{3, h}$, where $\left(\boldsymbol{v}_{1, h}, \phi_{1, h}\right) \in \stackrel{\circ}{\boldsymbol{V}}_{h} \times \hat{Q}_{h}$ satisfies

$$
\begin{gathered}
\left(\boldsymbol{v}_{1, h}, \boldsymbol{w}\right)_{\Omega_{h}}+\left(\phi_{1, h}, \operatorname{div} \boldsymbol{w}\right)_{\Omega_{h}}=0, \quad \boldsymbol{w} \in \boldsymbol{V}_{h}, \\
\left(\operatorname{div} \boldsymbol{v}_{1, h}, q_{h}\right)_{\Omega_{h}}=-\left(p-\hat{p}, q_{h}\right)_{\Omega_{h}} \quad q_{h} \in Q_{h} .
\end{gathered}
$$

$\left(\boldsymbol{v}_{2, h}, \phi_{2, h}\right) \in \boldsymbol{V}_{h} \times Q_{h}$ satisfies

$$
\begin{gathered}
\left(\boldsymbol{v}_{2, h}, \boldsymbol{w}\right)_{\Omega_{h}}+\left(\phi_{2, h}, \operatorname{div} \boldsymbol{w}\right)_{\Omega_{h}}-\langle\boldsymbol{w} \cdot \boldsymbol{n}, \lambda-\hat{\lambda}\rangle_{\Gamma_{h}}=0, \quad \boldsymbol{w} \in \boldsymbol{V}_{h}, \\
\left(\operatorname{div} \boldsymbol{v}_{2, h}, q\right)_{\Omega_{h}}=0, \quad q \in Q_{h},
\end{gathered}
$$

and $\left(\boldsymbol{v}_{3, h}, \phi_{3, h}\right) \in \boldsymbol{V}_{h} \times \hat{Q}_{h}$ satisfies

$$
\begin{gathered}
\left(\boldsymbol{v}_{3, h}, \boldsymbol{w}\right)_{\Omega_{h}}+\left(\phi_{3, h}, \operatorname{div} \boldsymbol{w}\right)_{\Omega_{h}}=0, \quad \boldsymbol{w} \in \stackrel{\circ}{\boldsymbol{V}}_{h}, \\
\left(\operatorname{div} \boldsymbol{v}_{3, h}, q_{h}\right)_{\Omega_{h}}=\frac{1}{\left|\Omega_{h}\right|}\left(\hat{\lambda}-\hat{p}, q_{h}\right)_{\Omega_{h}} \quad q_{h} \in Q_{h}, \\
\boldsymbol{v}_{3, h} \cdot \boldsymbol{n}=\frac{\hat{\lambda}-\hat{p}}{\left|\Gamma_{h}\right|} \text { on } \Gamma_{h} .
\end{gathered}
$$

Hypotheses (5.7) and (5.8) ensure the existence and uniqueness of $\boldsymbol{v}_{1, h}$ and $\boldsymbol{v}_{3, h}$, respectively, and also the a priori estimates

$$
\left\|\boldsymbol{v}_{1, h}\right\|_{H\left(\operatorname{div}, \Omega_{h}\right)} \leq C\|p-\hat{p}\|_{L^{2}\left(\Omega_{h}\right)}, \quad\left\|\boldsymbol{v}_{3, h}\right\|_{H\left(\operatorname{div}, \Omega_{h}\right)} \leq C|\hat{\lambda}-\hat{p}| .
$$

Then,

$$
\begin{array}{r}
-\left(p, \operatorname{div} \boldsymbol{v}_{h}\right)_{\Omega_{h}}+\left\langle\boldsymbol{v}_{h} \cdot \boldsymbol{n}, \lambda\right\rangle_{\Gamma_{h}}=(p, p-\hat{p})_{\Omega_{h}}-\frac{1}{\left|\Omega_{h}\right|}(p, \hat{\lambda}-\hat{p})_{\Omega_{h}} \\
+\frac{1}{\left|\Gamma_{h}\right|}\langle\lambda, \hat{\lambda}-\hat{p}\rangle_{\Gamma_{h}}+\left\langle\boldsymbol{v}_{2, h} \cdot \boldsymbol{n}, \lambda\right\rangle_{\Gamma_{h}}=\|p-\hat{p}\|_{L^{2}\left(\Omega_{h}\right)}^{2}+|\hat{\lambda}-\hat{p}|^{2}+\left\langle\boldsymbol{v}_{2, h} \cdot \boldsymbol{n}, \lambda\right\rangle_{\Gamma_{h}} \\
=\|p-\hat{p}\|_{L^{2}\left(\Omega_{h}\right)}^{2}+|\hat{\lambda}-\hat{p}|^{2}+\left\langle\boldsymbol{v}_{2, h} \cdot \boldsymbol{n}, \lambda-\hat{\lambda}\right\rangle_{\Gamma_{h}} . \\
=\|p-\hat{p}\|_{L^{2}\left(\Omega_{h}\right)}^{2}+|\hat{\lambda}-\hat{p}|^{2}+\left\|\boldsymbol{v}_{2, h}\right\|_{L^{2}\left(\Omega_{h}\right)}^{2} .
\end{array}
$$

Hypothesis (5.8) implies we can find $\boldsymbol{w} \in \boldsymbol{V}_{h}$ such that

$$
\operatorname{div} \boldsymbol{w}=\phi_{2, h} \text { in } \Omega_{h}, \quad \boldsymbol{w} \cdot \boldsymbol{n}=\frac{1}{\left|\Gamma_{h}\right|} \int_{\Omega_{h}} \phi_{2, h} d x \quad \text { on } \Gamma_{h}, \quad\|\boldsymbol{w}\|_{H(\operatorname{div}, \Omega)} \leq \gamma\left\|\phi_{2, h}\right\|_{L^{2}\left(\Omega_{h}\right)} .
$$


Using (5.12), and applying simple estimates, we get

$$
\left\|\phi_{2, h}\right\|_{L^{2}\left(\Omega_{h}\right)} \leq \gamma\left\|\boldsymbol{v}_{2, h}\right\|_{L^{2}\left(\Omega_{h}\right)}
$$

and so, by (5.9), (5.10), we get

$$
|\lambda-\hat{\lambda}|_{H_{h}^{1 / 2}\left(\Gamma_{h}\right)} \leq\left(\left\|\boldsymbol{v}_{2, h}\right\|_{L^{2}\left(\Omega_{h}\right)}^{2}+\left\|\phi_{2, h}\right\|_{L^{2}\left(\Omega_{h}\right)}^{2}\right)^{1 / 2} \leq\left(1+\gamma^{2}\right)^{1 / 2}\left\|\boldsymbol{v}_{2, h}\right\|_{L^{2}\left(\Omega_{h}\right)} .
$$

Combining these results, we have

$$
\begin{aligned}
-\left(p, \operatorname{div} \boldsymbol{v}_{h}\right)_{\Omega_{h}}+\left\langle\boldsymbol{v}_{h} \cdot \boldsymbol{n}, \lambda\right\rangle_{\Gamma_{h}}=\|p-\hat{p}\|_{L^{2}\left(\Omega_{h}\right)}^{2} & +|\hat{\lambda}-\hat{p}|^{2}+\left\|\boldsymbol{v}_{2, h}\right\|_{L^{2}\left(\Omega_{h}\right)}^{2} \\
& \geq\|p-\hat{p}\|_{L^{2}\left(\Omega_{h}\right)}^{2}+|\hat{\lambda}-\hat{p}|^{2}+\left(1+\gamma^{2}\right)^{-1}|\lambda-\hat{\lambda}|_{H_{h}^{1 / 2}\left(\Gamma_{h}\right)}^{2} .
\end{aligned}
$$

Finally, we need an upper bound:

$$
\left\|\boldsymbol{v}_{h}\right\|_{H\left(\mathrm{div}, \Omega_{h}\right)} \leq\left\|\boldsymbol{v}_{1, h}\right\|_{H\left(\mathrm{div}, \Omega_{h}\right)}+\left\|\boldsymbol{v}_{2, h}\right\|_{L^{2}\left(\Omega_{h}\right)}+\left\|\boldsymbol{v}_{3, h}\right\|_{H\left(\mathrm{div}, \Omega_{h}\right)} .
$$

Since $\operatorname{div} \boldsymbol{v}_{2, h}=\mathbf{0}$, to obtain a bound on $\left\|\boldsymbol{v}_{2, h}\right\|_{H\left(\operatorname{div}, \Omega_{h}\right)}=\left\|\boldsymbol{v}_{2, h}\right\|_{L^{2}\left(\Omega_{h}\right)}$, we choose $\boldsymbol{w}=\boldsymbol{v}_{2, h}$ in $(5.12)$ to get

$$
\left\|\boldsymbol{v}_{2, h}\right\|_{L^{2}\left(\Omega_{h}\right)}^{2}=\left\langle\boldsymbol{v}_{2, h} \cdot \boldsymbol{n}, \lambda-\hat{\lambda}\right\rangle_{\Gamma_{h}} \leq|\lambda-\hat{\lambda}|_{H_{h}^{1 / 2}\left(\Gamma_{h}\right)}\left\|\boldsymbol{v}_{2, h}\right\|_{L^{2}\left(\Omega_{h}\right)} .
$$

Hence,

$$
\left\|\boldsymbol{v}_{2, h}\right\|_{L^{2}\left(\Omega_{h}\right)} \leq|\lambda-\hat{\lambda}|_{H_{h}^{1 / 2}\left(\Gamma_{h}\right)} .
$$

Inserting the bounds on $\left\|\boldsymbol{v}_{1, h}\right\|_{H\left(\operatorname{div}, \Omega_{h}\right)}$ and $\left\|\boldsymbol{v}_{3, h}\right\|_{H\left(\operatorname{div}, \Omega_{h}\right)}$ given above, we have

$$
\left\|\boldsymbol{v}_{h}\right\|_{H\left(\operatorname{div}, \Omega_{h}\right)} \leq C\left(\|p-\hat{p}\|_{L^{2}\left(\Omega_{h}\right)}+|\hat{\lambda}-\hat{p}|+|\lambda-\hat{\lambda}|_{H_{h}^{1 / 2}\left(\Gamma_{h}\right)}\right) .
$$

Next, set $\boldsymbol{Y}=-\boldsymbol{Y}_{0, h}$, where $\boldsymbol{Y}_{0, h}$ is defined by (5.11). Then

$$
-\frac{1}{\Delta t}\langle\lambda \boldsymbol{n}, \boldsymbol{Y}\rangle_{\Gamma_{h}}=\frac{1}{\Delta t}\left|\boldsymbol{Y}_{0, h}\right|_{H_{*}^{1}\left(\Gamma_{h}\right)}^{2}=\frac{1}{\Delta t}|\lambda|_{*, h, \Gamma_{h}}^{2} .
$$

Combining these results, we have

$$
\frac{b_{h}(p, \lambda ; \boldsymbol{v}, \boldsymbol{Y})}{\|(\boldsymbol{v}, \boldsymbol{Y})\|_{\boldsymbol{\Sigma}_{h}}} \geq C \frac{\|p-\hat{p}\|_{L^{2}\left(\Omega_{h}\right)}^{2}+|\lambda-\hat{\lambda}|_{H_{h}^{1 / 2}\left(\Gamma_{h}\right)}^{2}+|\hat{\lambda}-\hat{p}|^{2}+(1 / \Delta t)|\lambda|_{*, h, \Gamma_{h}}^{2}}{\left.\left[\left(\|p-\hat{p}\|_{L^{2}\left(\Omega_{h}\right)}^{2}+|\lambda-\hat{\lambda}|_{H_{h}^{1 / 2}\left(\Gamma_{h}\right)}^{2}+|\hat{\lambda}-\hat{p}|^{2}\right)+(1 / \Delta t)|\lambda|_{*, h, \Gamma_{h}}^{2}\right]\right]^{1 / 2}} \geq C\|(p, \lambda)\|_{\Theta_{h}} .
$$

The assertion follows by taking the supremum.

\section{Error Estimates}

In this section, we derive error estimates in a much simplified situation. More specifically, we obtain an upper bound on the error over one time step, assuming that $\Omega=\Omega_{h}$ is a polygonal domain and that $\boldsymbol{U}_{h}=\boldsymbol{U}_{\text {. }}$ Thus, we ignore the approximation of the domain and also the error accumulated over previous time steps due to time discretization and space discretization of the domain. Clearly, the result desired is a full error analysis over all time steps. The fact that the domain is changing makes this a challenging problem, which we hope to address in future work.

Let $\mathcal{T}_{h}$ denote a quasi-uniform triangulation of $\Omega$ consisting of triangles of maximum size $h$. The error estimates derived in this section will be for a special choice of finite dimensional spaces. Let $\boldsymbol{V}_{h}=\boldsymbol{B D}_{\boldsymbol{D}} \subset$ $H(\operatorname{div}, \Omega)$, the lowest order Brezzi-Douglas-Marini space of piecewise linear vector functions, and $Q_{h}$ be the set of piecewise constants. It is well-known that these spaces satisfy hypothesis (5.7). Hypothesis (5.8) is easily satisfied by first finding $\boldsymbol{w} \in\left[H^{1}(\Omega)\right]^{2}$ satisfying the equations of (5.8) and then choosing $\boldsymbol{w}_{h}$ to be the canonical interpolant of $\boldsymbol{w}$ in $\boldsymbol{B} \boldsymbol{D} \boldsymbol{M}_{1}$, i.e., on each edge $e$ of the triangulation, $\boldsymbol{w}_{h}$ has zero and first order moments agreeing with those of $\boldsymbol{w}$. Finally, choose $\Lambda_{h}$ and each of the two components of the space $\Upsilon_{h}$ to be the space of continuous piecewise linear functions defined with respect to the mesh obtained by the restriction of $\mathcal{T}_{h}$ to $\Gamma$. 
Remarks. The method developed by [40] required the use of an $H^{1}$ conforming velocity space (i.e., continuous piecewise quadratic for velocity). This requires more degrees-of-freedom to represent the vector velocity field than the $\boldsymbol{B D} \boldsymbol{M}_{1}$ space used here. Also, it is not the "natural" finite element space to use, since the velocity is only in $H(\operatorname{div}, \Omega)$. Note: the simulation results for the two methods look qualitatively very similar.

To obtain error estimates, we will make use of results on a continuous approximation of the discontinuous normal vector to a polygonal domain. These are presented below.

6.1. Continuous Normal Vector on Discrete Domains. Let $\Gamma_{h}$ be a polygonal (oriented) curve with an ordered set of vertices $\left\{\boldsymbol{x}_{i}\right\}_{i=1}^{N}$ that interpolates a $C^{2}$ curve $\Gamma$ (i.e., the true boundary). Denote by $E_{i}^{+} \subset \Gamma_{h}$ the edge segment with (ordered) end points $\left(\boldsymbol{x}_{i}, \boldsymbol{x}_{i+1}\right)$; similarly, $E_{i}^{-} \subset \Gamma_{h}$ corresponds to the edge segment $\left(\boldsymbol{x}_{i-1}, \boldsymbol{x}_{i}\right)$. The obvious considerations are made at the end points of an open polygonal curve; likewise for a closed curve.

For a polygonal domain, the unit normal vector is discontinuous (piecewise constant). However, we have the following result [38].

Lemma 6.1. Let $\hat{\Gamma}$ be a $C^{2}$ regular closed curve with approximating polygon $\Gamma_{h}$, where $h$ denotes the maximum length of all edges of the polygon. Suppose that $\Gamma_{h}$ approximates $\hat{\Gamma}$ such that $\hat{\Gamma}$ can be represented as the graph of a function $f$ near the edge $E_{k}^{+} \subset \Gamma_{h}$. Hence, the derivative is bounded by a constant (i.e., $\left.\left|f^{\prime}\right| \leq C_{f}\right)$. Then, the following estimates are true:

$$
\left|\boldsymbol{n}_{k}^{+}-\hat{\boldsymbol{n}}_{k+1}\right| \leq C_{0} h_{k}\left|f^{\prime \prime}\right|_{\infty, E_{k}^{+}} \leq C_{1} h_{k} \kappa_{k}, \quad\left|\boldsymbol{n}_{k}^{+}-\hat{\boldsymbol{n}}_{k}\right| \leq C_{0} h_{k}\left|f^{\prime \prime}\right|_{\infty, E_{k}^{+}} \leq C_{1} h_{k} \kappa_{k},
$$

where $\hat{\boldsymbol{n}}_{k}$ and $\hat{\boldsymbol{n}}_{k+1}$ are the unit normals of $\hat{\Gamma}$ at the points $\boldsymbol{x}_{k}$ and $\boldsymbol{x}_{k+1}$, respectively, $\boldsymbol{n}_{k}^{+}$is the unit normal vector of $E_{k}^{+}, h_{k}$ is the length of $E_{k}^{+}, \kappa_{k}$ is the maximum curvature of the portion of $\hat{\Gamma}$ between $\boldsymbol{x}_{k}$ and $\boldsymbol{x}_{k+1}$, and $C_{0}$ and $C_{1}$ are constants independent of $h_{k}$.

Using Lemma 6.1, we can establish the following results.

Lemma 6.2. Let $\Gamma_{h} \subset \mathbb{R}^{2}$ be a polygonal curve (open or closed) and let $\boldsymbol{n}$ be the piecewise constant normal vector of $\Gamma_{h}$. Assume that $\Gamma_{h}$ interpolates a smooth $C^{2}$ curve $\Gamma$. Then there exists a vector field $\boldsymbol{n}_{s}$ that satisfies the following properties:

(1) $\boldsymbol{n}_{s}$ is continuous and piecewise linear on $\Gamma_{h}$.

(2) $\boldsymbol{n}_{s} \cdot \boldsymbol{n}=1$ everywhere on $\Gamma_{h}$.

(3) $\left\|\boldsymbol{n}_{s}\right\|_{\boldsymbol{H}^{1}\left(\Gamma_{h}\right)} \leq K$, where $K>0$ is independent of $h$, depending only on the curvature of $\Gamma$.

Remarks. The assumption that there exists a smooth curve $\Gamma$ that $\Gamma_{h}$ interpolates is easily satisfied by taking a cubic spline interpolant ( $a C^{2}$ curve) whose "knots" coincide with the vertices of the polygon $\Gamma_{h}$.

Proof. Let $\left\{\phi_{i}\right\}_{i=1}^{N}$ be the set of continuous piecewise linear 'hat' functions defined on $\Gamma_{h}$, i.e.,

$$
\phi_{i}\left(\boldsymbol{x}_{j}\right)= \begin{cases}1, & i=j, \\ 0, & i \neq j .\end{cases}
$$

The continuous vector field $\boldsymbol{n}_{s}$ is given by an explicit construction:

$$
\boldsymbol{n}_{s}:=\sum_{i=1}^{N} \frac{\boldsymbol{n}_{i}^{+}+\boldsymbol{n}_{i}^{-}}{1+\boldsymbol{n}_{i}^{+} \cdot \boldsymbol{n}_{i}^{-}} \phi_{i} .
$$

Note $\boldsymbol{n}_{s}$ is bounded in $L^{\infty}\left(\Gamma_{h}\right)$ as long as the angles of the polygon at the vertices are strictly bounded away from $0^{\circ}$ and $360^{\circ}$. 
Property (1) is satisfied because the set $\left\{\phi_{i}\right\}_{i=1}^{N}$ is a continuous basis. To prove property (2), we compute $\boldsymbol{n}_{s} \cdot \boldsymbol{n}$ on the "left" and "right" edges of each vertex:

$$
\begin{aligned}
& \boldsymbol{n}_{s}\left(\boldsymbol{x}_{k}^{-}\right) \cdot \boldsymbol{n}\left(\boldsymbol{x}_{k}^{-}\right)=\boldsymbol{n}\left(\boldsymbol{x}_{k}^{-}\right) \cdot \sum_{i=1}^{N} \frac{\boldsymbol{n}_{i}^{+}+\boldsymbol{n}_{i}^{-}}{1+\boldsymbol{n}_{i}^{+} \cdot \boldsymbol{n}_{i}^{-}} \phi_{i}\left(\boldsymbol{x}_{k}^{-}\right)=\frac{\boldsymbol{n}_{k}^{-} \cdot\left(\boldsymbol{n}_{k}^{+}+\boldsymbol{n}_{k}^{-}\right)}{1+\boldsymbol{n}_{k}^{+} \cdot \boldsymbol{n}_{k}^{-}}=1 . \\
& \boldsymbol{n}_{s}\left(\boldsymbol{x}_{k}^{+}\right) \cdot \boldsymbol{n}\left(\boldsymbol{x}_{k}^{+}\right)=\boldsymbol{n}\left(\boldsymbol{x}_{k}^{+}\right) \cdot \sum_{i=1}^{N} \frac{\boldsymbol{n}_{i}^{+}+\boldsymbol{n}_{i}^{-}}{1+\boldsymbol{n}_{i}^{+} \cdot \boldsymbol{n}_{i}^{-}} \phi_{i}\left(\boldsymbol{x}_{k}^{+}\right)=\frac{\boldsymbol{n}_{k}^{+} \cdot\left(\boldsymbol{n}_{k}^{+}+\boldsymbol{n}_{k}^{-}\right)}{1+\boldsymbol{n}_{k}^{+} \cdot \boldsymbol{n}_{k}^{-}}=1 .
\end{aligned}
$$

Ergo, $\left(\boldsymbol{n}_{s} \cdot \boldsymbol{n}\right)\left(\boldsymbol{x}_{k}\right)=1$, for $1 \leq k \leq N$. Since $\boldsymbol{n}_{s} \cdot \boldsymbol{n}$ is linear on each edge segment of $\Gamma_{h}$, then $\boldsymbol{n}_{s} \cdot \boldsymbol{n}=1$ everywhere on $\Gamma_{h}$, which proves property $(2)$.

Property (3) can be easily verified using Lemma 6.1.

6.2. Error estimates over a single time step. To derive an error estimate, we first define some standard projection operators for the spaces $\boldsymbol{V}_{h}$ and $Q_{h}$ and discuss their approximation properties. We let $\boldsymbol{u}_{I}$ be the canonical projection of $\boldsymbol{u}$ into $\boldsymbol{B} \boldsymbol{D} \boldsymbol{M}_{1}, p_{I}$ the $L^{2}$ projection of $p$ into $Q_{h}, \lambda_{I}$ the $L^{2}$ projection of $\lambda$ into $\Lambda_{h}$, and $W_{I}$ the continuous, piecewise, linear interpolant of $\boldsymbol{W}$ in $\boldsymbol{\Upsilon}_{h}$. Note that $\boldsymbol{u}_{I}$ and $p_{I}$ satisfy

$$
\int_{e}\left[\boldsymbol{u}-\boldsymbol{u}_{I}\right] \cdot \boldsymbol{n} r d s=0, \quad r \in \operatorname{span}(1, s), \quad \int_{T}\left[p-p_{I}\right] d x=0,
$$

for each edge $e$ and triangle $T$ of $\mathcal{T}_{h}$. For $\boldsymbol{u}$ in $\left[H^{1}(\Omega)\right]^{2}$, we have the standard estimate

$$
\left\|\boldsymbol{u}-\boldsymbol{u}_{I}\right\|_{L^{2}(\Omega)} \leq C h\|\boldsymbol{u}\|_{H^{1}(\Omega)} .
$$

To obtain error estimates with minimal regularity assumptions, we need a non-standard estimate for the error $\left\|\boldsymbol{u}-\boldsymbol{u}_{I}\right\|_{L^{2}(\Omega)}$, which we now derive.

Lemma 6.3. Suppose for some $p>2$ and $0<s \leq 1, \boldsymbol{u} \in\left[L^{p}(\Omega) \cap W_{q}^{s}(\Omega)\right]^{2}$, where $q=2 p /(2+s(p-2))$. If $\operatorname{div} \boldsymbol{u}=0$, then

$$
\left\|\boldsymbol{u}-\boldsymbol{u}_{I}\right\|_{L^{2}(\Omega)} \leq C h^{s}\|\boldsymbol{u}\|_{W_{q}^{s}(\Omega)} .
$$

Proof. Since $\operatorname{div} \boldsymbol{u}=0$ and $\boldsymbol{u} \in L^{p}(\Omega)$, we have $\boldsymbol{u}=\operatorname{curl} z$ for some $z$ in $W^{1, p}(\Omega)$. Let $\boldsymbol{z}_{I}$ be an interpolant of $\boldsymbol{z}$ in the space of continuous piecewise quadratics defined by $z_{I}-z=0$ at the vertices of the triangulation and the average value of $z_{I}-z=0$ on each edge of the triangulation. It is then easy to check that $\boldsymbol{u}_{I}$, the $\boldsymbol{B} \boldsymbol{D} \boldsymbol{M}_{1}$ interpolant of $\boldsymbol{u}$ defined above, satisfies $\boldsymbol{u}_{I}=\operatorname{curl} \boldsymbol{z}_{I}$. Hence, using standard estimates (c.f. [12], p. 124), for $p>2$,

$$
\left\|\boldsymbol{u}-\boldsymbol{u}_{I}\right\|_{L^{2}(\Omega)} \leq\left\|\boldsymbol{u}-\boldsymbol{u}_{I}\right\|_{L^{p}(\Omega)}=\left\|\operatorname{curl}\left(z-z_{I}\right)\right\|_{L^{p}(\Omega)} \leq C\|\operatorname{curl} z\|_{L^{p}(\Omega)}=C\|\boldsymbol{u}\|_{L^{p}(\Omega)} .
$$

We can now interpolate between the spaces $L^{p}$ and $H^{1}$ to get an error estimate with less regularity. From Theorem 6.4.5 of [6], we have

$$
\left[W_{p_{0}}^{s_{0}}, W_{p_{1}}^{s_{1}}\right]_{[\theta]}=W_{q}^{s}, \quad s_{0} \neq s_{1}, \quad 1<p_{0}, p_{1}<\infty,
$$

where

$$
s=(1-\theta) s_{0}+\theta s_{1}, \quad \frac{1}{q}=\frac{1-\theta}{p_{0}}+\frac{\theta}{p_{1}} .
$$

In our case, $s_{0}=0, s_{1}=1, p_{0}=p$, and $p_{1}=2$. So $s=\theta$, and $q=2 p /[2+s(p-2)]$. Finally, we can combine (6.1) and (6.2) (c.f. [9], p. 115) to get

$$
\left\|\boldsymbol{u}-\boldsymbol{u}_{I}\right\|_{L^{2}(\Omega)} \leq C h^{s}\|\boldsymbol{u}\|_{W_{q}^{s}(\Omega)}, \quad 0<s \leq 1,
$$

where we note that for $s=1, q=2$.

We now turn to the statement and proof of our basic error estimate. 
Theorem 6.4. Let $(\boldsymbol{u}, \boldsymbol{W}) \in \Sigma$ and $(p, \lambda) \in \Theta$ be the solution of (4.6)-(4.7) and $\left(\boldsymbol{u}_{h}, \boldsymbol{W}_{h}\right) \in \Sigma_{h}$ and $\left(p_{h}, \lambda_{h}\right) \in \Theta_{h}$ be the solution of (5.5)-(5.6). Then

$$
\begin{aligned}
\left\|\boldsymbol{u}_{h}-\boldsymbol{u}_{I}\right\|_{L^{2}(\Omega)}^{2}+\left|\nabla_{\Gamma}\left[\boldsymbol{W}_{h}-\boldsymbol{W}_{I}\right]\right|_{L^{2}(\Gamma)}^{2} & \leq C\left(\left\|\boldsymbol{u}-\boldsymbol{u}_{I}\right\|_{L^{2}(\Omega)}^{2}+\left|\nabla_{\Gamma}\left[\boldsymbol{W}-\boldsymbol{W}_{I}\right]\right|_{L^{2}(\Gamma)}^{2}\right. \\
& \left.+h^{-2}\left|\boldsymbol{W}-\boldsymbol{W}_{I}\right|_{L^{2}(\Gamma)}^{2}+(\Delta t)^{2}\left|\lambda-\lambda_{I}\right|_{H^{1 / 2}(\Gamma)}^{2}+h^{2}\left|\lambda-\lambda_{I}\right|_{L^{2}(\Gamma)}^{2}\right) .
\end{aligned}
$$

Proof. Combining the continuous and discrete equations, we get the error equations

$$
\begin{aligned}
a\left(\boldsymbol{u}_{h}-\boldsymbol{u}_{I}, \boldsymbol{W}_{h}-\boldsymbol{W}_{I} ; \boldsymbol{v}, \boldsymbol{Y}\right)+ & b\left(p_{h}-p_{I}, \lambda_{h}-\lambda_{I} ; \boldsymbol{v}, \boldsymbol{Y}\right) \\
& =a\left(\boldsymbol{u}-\boldsymbol{u}_{I}, \boldsymbol{W}-\boldsymbol{W}_{I} ; \boldsymbol{v}, \boldsymbol{Y}\right)+b\left(p-p_{I}, \lambda-\lambda_{I} ; \boldsymbol{v}, \boldsymbol{Y}\right), \\
b\left(q, \mu ; \boldsymbol{u}_{h}-\boldsymbol{u}_{I}, \boldsymbol{W}_{h}-\boldsymbol{W}_{I}\right) & =b\left(q, \mu ; \boldsymbol{u}-\boldsymbol{u}_{I}, \boldsymbol{W}-\boldsymbol{W}_{I}\right),
\end{aligned}
$$

for all $(\boldsymbol{v}, \boldsymbol{Y}) \in \boldsymbol{V}_{h} \times \boldsymbol{\Upsilon}_{h}$ and all $(q, \mu) \in Q_{h} \times \Lambda_{h}$. Choosing $\tilde{\boldsymbol{v}}=\boldsymbol{u}_{h}-\boldsymbol{u}_{I}, \tilde{\boldsymbol{Y}}=\boldsymbol{W}_{h}-\boldsymbol{W}_{I}, \tilde{q}=p_{h}-p_{I}$, and $\tilde{\mu}=\lambda_{h}-\lambda_{I}$, with $\boldsymbol{u}_{I}, p_{I}, \boldsymbol{W}_{I}$ and $\lambda_{I}$ defined above, and taking the difference of the error equations, we have

$$
\begin{aligned}
a\left(\boldsymbol{u}_{h}-\boldsymbol{u}_{I}, \boldsymbol{W}_{h}-\boldsymbol{W}_{I} ; \tilde{\boldsymbol{v}}, \tilde{\boldsymbol{Y}}\right)=a\left(\boldsymbol{u}-\boldsymbol{u}_{I}, \boldsymbol{W}-\boldsymbol{W}_{I} ; \tilde{\boldsymbol{v}}, \tilde{\boldsymbol{Y}}\right) \\
+b\left(p-p_{I}, \lambda-\lambda_{I} ; \tilde{\boldsymbol{v}}, \tilde{\boldsymbol{Y}}\right)-b\left(\tilde{q}, \tilde{\mu} ; \boldsymbol{u}-\boldsymbol{u}_{I}, \boldsymbol{W}-\boldsymbol{W}_{I}\right) .
\end{aligned}
$$

Hence,

$$
\begin{aligned}
\left(\frac{\alpha}{\Delta t}+\beta\right) \| \boldsymbol{u}_{h} & -\boldsymbol{u}_{I} \|_{L^{2}(\Omega)}^{2}+\frac{1}{\Delta t}\left|\nabla_{\Gamma}\left[\boldsymbol{W}_{h}-\boldsymbol{W}_{I}\right]\right|_{L^{2}(\Gamma)}^{2} \\
& =\left(\frac{\alpha}{\Delta t}+\beta\right)\left(\boldsymbol{u}-\boldsymbol{u}_{I}, \boldsymbol{u}_{h}-\boldsymbol{u}_{I}\right)_{\Omega}+\frac{1}{\Delta t}\left\langle\nabla_{\Gamma}\left[\boldsymbol{W}-\boldsymbol{W}_{I}\right], \mid \nabla_{\Gamma}\left[\boldsymbol{W}_{h}-\boldsymbol{W}_{I}\right]\right\rangle_{\Gamma} \\
-(p & \left.-p_{I}, \operatorname{div}\left[\boldsymbol{u}_{h}-\boldsymbol{u}_{I}\right]\right)_{\Omega}+\left\langle\left[\boldsymbol{u}_{h}-\boldsymbol{u}_{I}\right] \cdot \boldsymbol{n}, \lambda-\lambda_{I}\right\rangle_{\Gamma}-\frac{1}{\Delta t}\left\langle\lambda-\lambda_{I}, \boldsymbol{n} \cdot\left[\boldsymbol{W}_{h}-\boldsymbol{W}_{I}\right]\right\rangle_{\Gamma} \\
- & {\left[-\left(p_{h}-p_{I}, \operatorname{div}\left[\boldsymbol{u}-\boldsymbol{u}_{I}\right]\right)_{\Omega}+\left\langle\left[\boldsymbol{u}-\boldsymbol{u}_{I}\right] \cdot \boldsymbol{n}, \lambda_{h}-\lambda_{I}\right\rangle_{\Gamma}-\frac{1}{\Delta t}\left\langle\lambda_{h}-\lambda_{I}, \boldsymbol{n} \cdot\left[\boldsymbol{W}-\boldsymbol{W}_{I}\right]\right\rangle_{\Gamma}\right] . }
\end{aligned}
$$

From the definitions of $\boldsymbol{u}_{I}$ and $p_{I}$, we have

$\left(q_{h}, \operatorname{div}\left[\boldsymbol{u}-\boldsymbol{u}_{I}\right]\right)_{\Omega}=0, q_{h} \in Q_{h}, \quad\left(p-p_{I}, \operatorname{div} \boldsymbol{v}_{h}\right)_{\Omega}=0, \boldsymbol{v}_{h} \in \boldsymbol{V}_{h}, \quad\left\langle\left[\boldsymbol{u}-\boldsymbol{u}_{I}\right] \cdot \boldsymbol{n}, \mu_{h}\right\rangle_{\Gamma}=0, \mu_{h} \in \Lambda_{h}$.

Using this result, we can simplify the previous identity and obtain

$$
\begin{aligned}
\left(\frac{\alpha}{\Delta t}+\beta\right) & \left\|\boldsymbol{u}_{h}-\boldsymbol{u}_{I}\right\|_{L^{2}(\Omega)}^{2}+\frac{1}{\Delta t}\left|\nabla_{\Gamma}\left[\boldsymbol{W}_{h}-\boldsymbol{W}_{I}\right]\right|_{L^{2}(\Gamma)}^{2} \\
= & \left(\frac{\alpha}{\Delta t}+\beta\right)\left(\boldsymbol{u}-\boldsymbol{u}_{I}, \boldsymbol{u}_{h}-\boldsymbol{u}_{I}\right)_{\Omega}+\frac{1}{\Delta t}\left\langle\nabla_{\Gamma}\left[\boldsymbol{W}-\boldsymbol{W}_{I}\right], \mid \nabla_{\Gamma}\left[\boldsymbol{W}_{h}-\boldsymbol{W}_{I}\right]\right\rangle_{\Gamma} \\
+ & \left\langle\lambda-\lambda_{I},\left[\boldsymbol{u}_{h}-\boldsymbol{u}_{I}\right] \cdot \boldsymbol{n}\right\rangle_{\Gamma}-\frac{1}{\Delta t}\left\langle\lambda-\lambda_{I}, \boldsymbol{n} \cdot\left[\boldsymbol{W}_{h}-\boldsymbol{W}_{I}\right]\right\rangle_{\Gamma}+\frac{1}{\Delta t}\left\langle\lambda_{h}-\lambda_{I}, \boldsymbol{n} \cdot\left[\boldsymbol{W}-\boldsymbol{W}_{I}\right]\right\rangle_{\Gamma} .
\end{aligned}
$$

Using the Schwarz inequality, we can bound the first two terms.

$$
\begin{gathered}
\left(\boldsymbol{u}-\boldsymbol{u}_{I}, \boldsymbol{u}_{h}-\boldsymbol{u}_{I}\right)_{\Omega} \leq\left\|\boldsymbol{u}-\boldsymbol{u}_{I}\right\|_{L^{2}(\Omega)}\left\|\boldsymbol{u}_{h}-\boldsymbol{u}_{I}\right\|_{L^{2}(\Omega)}, \\
\left\langle\nabla_{\Gamma}\left[\boldsymbol{W}-\boldsymbol{W}_{I}\right], \mid \nabla_{\Gamma}\left[\boldsymbol{W}_{h}-\boldsymbol{W}_{I}\right]\right\rangle_{\Gamma} \leq\left|\nabla_{\Gamma}\left[\boldsymbol{W}-\boldsymbol{W}_{I}\right]\right|_{L^{2}(\Gamma)}\left|\nabla_{\Gamma}\left[\boldsymbol{W}_{h}-\boldsymbol{W}_{I}\right]\right|_{L^{2}(\Gamma)} .
\end{gathered}
$$

Since $\operatorname{div}\left[\boldsymbol{u}_{h}-\boldsymbol{u}_{I}\right]=0$, we have

$$
\begin{aligned}
\left\langle\lambda-\lambda_{I},\left[\boldsymbol{u}_{h}-\boldsymbol{u}_{I}\right] \cdot \boldsymbol{n}\right\rangle_{\Gamma} \leq & \left|\lambda-\lambda_{I}\right|_{H^{1 / 2}(\Gamma)}\left|\left[\boldsymbol{u}_{h}-\boldsymbol{u}_{I}\right] \cdot \boldsymbol{n}\right|_{H^{-1 / 2}(\Gamma)} \\
& \leq C\left|\lambda-\lambda_{I}\right|_{H^{1 / 2}(\Gamma)}\left\|\boldsymbol{u}_{h}-\boldsymbol{u}_{I}\right\|_{H(\operatorname{div}, \Omega)} \leq C\left|\lambda-\lambda_{I}\right|_{H^{1 / 2}(\Gamma)}\left\|\boldsymbol{u}_{h}-\boldsymbol{u}_{I}\right\|_{L^{2}(\Omega)} .
\end{aligned}
$$

It thus remains to bound the terms $\left\langle\lambda-\lambda_{I}, \boldsymbol{n} \cdot\left[\boldsymbol{W}_{h}-\boldsymbol{W}_{I}\right]\right\rangle_{\Gamma}$ and $\left\langle\lambda_{h}-\lambda_{I}, \boldsymbol{n} \cdot\left[\boldsymbol{W}-\boldsymbol{W}_{I}\right]\right\rangle_{\Gamma}$. Since the derivation is somewhat lengthy, we state the results here and prove them in two lemmas following the theorem. We will show that 


$$
\begin{aligned}
& \left\langle\lambda-\lambda_{I}, \boldsymbol{n} \cdot\left[\boldsymbol{W}_{h}-\boldsymbol{W}_{I}\right]\right\rangle_{\Gamma} \\
\leq & C h\left|\lambda-\lambda_{I}\right|_{L^{2}(\Gamma)}\left(\left|\nabla_{\Gamma}\left[\boldsymbol{W}_{h}-\boldsymbol{W}_{I}\right]\right|_{L^{2}(\Gamma)}+\left|\boldsymbol{W}-\boldsymbol{W}_{I}\right|_{L^{2}(\Gamma)}+\Delta t\left\|\boldsymbol{u}_{h}-\boldsymbol{u}_{I}\right\|_{L^{2}(\Omega)}+\Delta t\left\|\boldsymbol{u}_{I}-\boldsymbol{u}\right\|_{L^{2}(\Omega)}\right)
\end{aligned}
$$

and

$$
\begin{aligned}
& \left\langle\lambda_{h}-\lambda_{I}, \boldsymbol{n} \cdot\left[\boldsymbol{W}-\boldsymbol{W}_{I}\right]\right\rangle_{\Gamma} \\
& \quad \leq C\left|\boldsymbol{W}-\boldsymbol{W}_{I}\right|_{L^{2}(\Gamma)}\left(\left|\lambda-\lambda_{I}\right|_{L^{2}(\Gamma)}+h^{-1}\left|\nabla_{\Gamma}\left[\boldsymbol{W}_{h}-\boldsymbol{W}_{I}\right]\right|_{L^{2}(\Gamma)}+h^{-1}\left|\nabla_{\Gamma}\left[\boldsymbol{W}_{I}-\boldsymbol{W}\right]\right|_{L^{2}(\Gamma)}\right) .
\end{aligned}
$$

Inserting (6.6), (6.7), (6.8), (6.9), and (6.10) in (6.5) gives

$$
\begin{gathered}
\left(\frac{\alpha}{\Delta t}+\beta\right)\left\|\boldsymbol{u}_{h}-\boldsymbol{u}_{I}\right\|_{L^{2}(\Omega)}^{2}+\frac{1}{\Delta t}\left|\nabla_{\Gamma}\left[\boldsymbol{W}_{h}-\boldsymbol{W}_{I}\right]\right|_{L^{2}(\Gamma)}^{2} \\
\leq\left(\frac{\alpha}{\Delta t}+\beta\right)\left\|\boldsymbol{u}-\boldsymbol{u}_{I}\right\|_{L^{2}(\Omega)}\left\|\boldsymbol{u}_{h}-\boldsymbol{u}_{I}\right\|_{L^{2}(\Omega)}+\frac{1}{\Delta t}\left|\nabla_{\Gamma}\left[\boldsymbol{W}-\boldsymbol{W}_{I}\right]\right|_{L^{2}(\Gamma)}\left|\nabla_{\Gamma}\left[\boldsymbol{W}_{h}-\boldsymbol{W}_{I}\right]\right|_{L^{2}(\Gamma)} \\
+\frac{C h}{\Delta t}\left|\lambda-\lambda_{I}\right|_{L^{2}(\Gamma)}\left\{\left|\nabla_{\Gamma}\left[\boldsymbol{W}_{h}-\boldsymbol{W}_{I}\right]\right|_{L^{2}(\Gamma)}+\left|\boldsymbol{W}-\boldsymbol{W}_{I}\right|_{L^{2}(\Gamma)}+\Delta t\left\|\boldsymbol{u}_{h}-\boldsymbol{u}_{I}\right\|_{L^{2}(\Omega)}+\Delta t\left\|\boldsymbol{u}_{I}-\boldsymbol{u}\right\|_{L^{2}(\Omega)}\right\} \\
\quad+C\left|\lambda-\lambda_{I}\right|_{H^{1 / 2}(\Gamma)}\left\|\boldsymbol{u}_{h}-\boldsymbol{u}_{I}\right\|_{L^{2}(\Omega)} \\
+\frac{C}{\Delta t}\left|\boldsymbol{W}-\boldsymbol{W}_{I}\right|_{L^{2}(\Gamma)}\left\{\left|\lambda-\lambda_{I}\right|_{L^{2}(\Gamma)}+h^{-1}\left|\nabla_{\Gamma}\left[\boldsymbol{W}_{h}-\boldsymbol{W}_{I}\right]\right|_{L^{2}(\Gamma)}+h^{-1}\left|\nabla_{\Gamma}\left[\boldsymbol{W}_{I}-\boldsymbol{W}\right]\right|_{L^{2}(\Gamma)}\right\} .
\end{gathered}
$$

Applying the arithmetic-geometric mean inequality and moving terms to the left-hand-side, we obtain

$$
\begin{aligned}
& \left(\frac{\alpha}{\Delta t}+\beta\right)\left\|\boldsymbol{u}_{h}-\boldsymbol{u}_{I}\right\|_{L^{2}(\Omega)}^{2}+\frac{1}{\Delta t}\left|\nabla_{\Gamma}\left[\boldsymbol{W}_{h}-\boldsymbol{W}_{I}\right]\right|_{L^{2}(\Gamma)}^{2} \\
& \leq C\left(\left(\frac{\alpha}{\Delta t}+\beta\right)\left\|\boldsymbol{u}-\boldsymbol{u}_{I}\right\|_{L^{2}(\Omega)}^{2}+\frac{1}{\Delta t}\left|\nabla_{\Gamma}\left[\boldsymbol{W}-\boldsymbol{W}_{I}\right]\right|_{L^{2}(\Gamma)}^{2}\right. \\
& \left.\quad+\Delta t\left|\lambda-\lambda_{I}\right|_{H^{1 / 2}(\Gamma)}^{2}+\frac{1}{\Delta t} h^{-2}\left|\boldsymbol{W}-\boldsymbol{W}_{I}\right|_{L^{2}(\Gamma)}^{2}+\frac{1}{\Delta t} h^{2}\left|\lambda-\lambda_{I}\right|_{L^{2}(\Gamma)}^{2}\right) .
\end{aligned}
$$

The proof is completed by multiplying the inequality by $\Delta t$.

Applying (6.3) and standard approximation theory estimates for our choice of finite dimensional spaces, we get the following order of convergence estimates.

Corollary 6.5. Let $(\boldsymbol{u}, \boldsymbol{W}) \in \Sigma$ and $(p, \lambda) \in \Theta$ be the solution of (4.6)-(4.7) and $\left(\boldsymbol{u}_{h}, \boldsymbol{W}_{h}\right) \in \Sigma_{h}$ and $\left(p_{h}, \lambda_{h}\right) \in \Theta_{h}$ be the solution of (5.5)-(5.6). If for some $0<s \leq 1$, and $q \geq 2, \boldsymbol{u} \in W_{q}^{s}(\Omega), \boldsymbol{W} \in H^{1+s}(\Gamma)$, $\lambda \in H^{1 / 2+s}(\Gamma)$, then

$$
\left\|\boldsymbol{u}-\boldsymbol{u}_{h}\right\|_{L^{2}(\Omega)}+\left|\nabla_{\Gamma}\left[\boldsymbol{W}-\boldsymbol{W}_{h}\right]\right|_{L^{2}(\Gamma)} \leq C h^{s}\left[\|\boldsymbol{u}\|_{W_{q}^{s}(\Omega)}+|\boldsymbol{W}|_{1+s, \Gamma}+\left(\Delta t+h^{3 / 2}\right)|\lambda|_{1 / 2+s, \Gamma}\right] .
$$

Proof. The result follows by applying Theorem 6.4 and the triangle inequality.

\section{Lemma 6.6.}

$$
\begin{aligned}
& \left\langle\lambda-\lambda_{I}, \boldsymbol{n} \cdot\left[\boldsymbol{W}_{h}-\boldsymbol{W}_{I}\right]\right\rangle_{\Gamma} \\
& \leq C h\left|\lambda-\lambda_{I}\right|_{L^{2}(\Gamma)}\left(\left|\nabla_{\Gamma}\left[\boldsymbol{W}_{h}-\boldsymbol{W}_{I}\right]\right|_{L^{2}(\Gamma)}+\left|\boldsymbol{W}-\boldsymbol{W}_{I}\right|_{L^{2}(\Gamma)}+\Delta t\left\|\boldsymbol{u}_{h}-\boldsymbol{u}_{I}\right\|_{L^{2}(\Omega)}+\Delta t\left\|\boldsymbol{u}_{I}-\boldsymbol{u}\right\|_{L^{2}(\Omega)}\right) .
\end{aligned}
$$

Proof. Since $\lambda_{I}$ is the $L^{2}$ projection of $\lambda$ into $\Lambda_{h}$, we get for all $\mu \in \Lambda_{h}$,

$$
\left\langle\lambda-\lambda_{I}, \boldsymbol{n} \cdot\left[\boldsymbol{W}_{h}-\boldsymbol{W}_{I}\right]\right\rangle_{\Gamma}=\left\langle\lambda-\lambda_{I}, \boldsymbol{n} \cdot\left[\boldsymbol{W}_{h}-\boldsymbol{W}_{I}\right]-\mu\right\rangle_{\Gamma} \leq\left|\lambda-\lambda_{I}\right|_{L^{2}(\Gamma)}\left|\boldsymbol{n} \cdot\left[\boldsymbol{W}_{h}-\boldsymbol{W}_{I}\right]-\mu\right|_{L^{2}(\Gamma)} \cdot
$$

We now choose $\mu=\sum_{i=1}^{N_{S}}\left[\boldsymbol{W}_{h}-\boldsymbol{W}_{I}\right]\left(\boldsymbol{x}_{i}\right) \cdot \hat{\boldsymbol{n}}_{i} \phi_{i}$, where $\hat{\boldsymbol{n}}_{i}$ is defined in Lemma 6.1. Then on the edge $E_{k}^{+}$, we have

$$
\boldsymbol{n} \cdot\left[\boldsymbol{W}_{h}-\boldsymbol{W}_{I}\right]-\mu=\left[\boldsymbol{n}_{k}^{+}-\hat{\boldsymbol{n}}_{k}\right] \cdot\left[\boldsymbol{W}_{h}-\boldsymbol{W}_{I}\right]\left(\boldsymbol{x}_{k}\right) \phi_{k}+\left[\boldsymbol{n}_{k}^{+}-\hat{\boldsymbol{n}}_{k+1}\right] \cdot\left[\boldsymbol{W}_{h}-\boldsymbol{W}_{I}\right]\left(x_{k+1}\right) \phi_{k+1} .
$$


By Lemma 6.1, we then obtain

$\left|\boldsymbol{n} \cdot\left[\boldsymbol{W}_{h}-\boldsymbol{W}_{I}\right]-\mu\right|_{L^{2}(\Gamma)} \leq \max _{k} \max \left(\left|\boldsymbol{n}_{k}^{+}-\hat{\boldsymbol{n}}_{k}\right|,\left|\boldsymbol{n}_{k}^{+}-\hat{\boldsymbol{n}}_{k+1}\right|\right)\left|\boldsymbol{W}_{h}-\boldsymbol{W}_{I}\right|_{L^{2}(\Gamma)} \leq C h\left(\max _{k} \kappa_{k}\right)\left|\boldsymbol{W}_{h}-\boldsymbol{W}_{I}\right|_{L^{2}(\Gamma)}$

By the proof of Proposition 4.1, we have $|\boldsymbol{W}|_{L^{2}(\Gamma)} \leq C|\boldsymbol{W}|_{H_{*}^{1}(\Gamma)}$. Hence, in order to complete our error estimates, we need to bound $\left|\left\langle\left[\boldsymbol{W}_{h}-\boldsymbol{W}_{I}\right] \cdot \boldsymbol{n}, \mu\right\rangle_{\Gamma}\right|$ for $\mu=x$ and $\mu=y$. Now

$$
\begin{aligned}
\frac{1}{\Delta t}\left\langle\left[\boldsymbol{W}_{h}-\boldsymbol{W}_{I}\right] \cdot \boldsymbol{n}, \mu\right\rangle_{\Gamma}=\frac{1}{\Delta t}\left\langle\left[\boldsymbol{W}_{h}-\boldsymbol{W}\right] \cdot \boldsymbol{n}, \mu\right\rangle_{\Gamma}+\frac{1}{\Delta t}\left\langle\left[\boldsymbol{W}-\boldsymbol{W}_{I}\right] \cdot \boldsymbol{n}, \mu\right\rangle_{\Gamma} \\
\quad=\left\langle\left[\boldsymbol{u}_{h}-\boldsymbol{u}\right] \cdot \boldsymbol{n}, \mu\right\rangle_{\Gamma}+\frac{1}{\Delta t}\left\langle\left[\boldsymbol{W}-\boldsymbol{W}_{I}\right] \cdot \boldsymbol{n}, \mu\right\rangle_{\Gamma}=\left(\boldsymbol{u}_{h}-\boldsymbol{u}, \nabla \mu\right)_{\Gamma}+\frac{1}{\Delta t}\left\langle\left[\boldsymbol{W}-\boldsymbol{W}_{I}\right] \cdot \boldsymbol{n}, \mu\right\rangle_{\Gamma} .
\end{aligned}
$$

Then for $\mu=x$ or $\mu=y$, we get

$$
\left|\left\langle\left[\boldsymbol{W}_{h}-\boldsymbol{W}_{I}\right] \cdot \boldsymbol{n}, \mu\right\rangle_{\Gamma}\right| \leq C\left(\left|\boldsymbol{W}-\boldsymbol{W}_{I}\right|_{L^{2}(\Gamma)}+\Delta t\left\|\boldsymbol{u}_{h}-\boldsymbol{u}_{I}\right\|_{L^{2}(\Omega)}+\Delta t\left\|\boldsymbol{u}_{I}-\boldsymbol{u}\right\|_{L^{2}(\Omega)}\right) .
$$

Hence,

$$
\left|\boldsymbol{W}_{h}-\boldsymbol{W}_{I}\right|_{L^{2}(\Gamma)} \leq C\left(\left|\nabla_{\Gamma}\left[\boldsymbol{W}_{h}-\boldsymbol{W}_{I}\right]\right|_{L^{2}(\Gamma)}+\left|\boldsymbol{W}-\boldsymbol{W}_{I}\right|_{L^{2}(\Gamma)}+\Delta t\left\|\boldsymbol{u}_{h}-\boldsymbol{u}_{I}\right\|_{L^{2}(\Omega)}+\Delta t\left\|\boldsymbol{u}_{I}-\boldsymbol{u}\right\|_{L^{2}(\Omega)}\right) .
$$

Combining these results, we get

$$
\begin{aligned}
& \left\langle\lambda-\lambda_{I}, \boldsymbol{n} \cdot\left[\boldsymbol{W}_{h}-\boldsymbol{W}_{I}\right]\right\rangle_{\Gamma} \\
& \leq C h\left|\lambda-\lambda_{I}\right|_{L^{2}(\Gamma)}\left(\left|\nabla_{\Gamma}\left[\boldsymbol{W}_{h}-\boldsymbol{W}_{I}\right]\right|_{L^{2}(\Gamma)}+\left|\boldsymbol{W}-\boldsymbol{W}_{I}\right|_{L^{2}(\Gamma)}+\Delta t\left\|\boldsymbol{u}_{h}-\boldsymbol{u}_{I}\right\|_{L^{2}(\Omega)}+\Delta t\left\|\boldsymbol{u}_{I}-\boldsymbol{u}\right\|_{L^{2}(\Omega)}\right) .
\end{aligned}
$$

\section{Lemma 6.7.}

$\left\langle\lambda_{h}-\lambda_{I}, \boldsymbol{n} \cdot\left[\boldsymbol{W}-\boldsymbol{W}_{I}\right]\right\rangle_{\Gamma} \leq C\left|\boldsymbol{W}-\boldsymbol{W}_{I}\right|_{L^{2}(\Gamma)}\left(\left|\lambda-\lambda_{I}\right|_{L^{2}(\Gamma)}+h^{-1}\left|\nabla_{\Gamma}\left[\boldsymbol{W}_{h}-\boldsymbol{W}_{I}\right]\right|_{L^{2}(\Gamma)}+h^{-1}\left|\nabla_{\Gamma}\left[\boldsymbol{W}_{I}-\boldsymbol{W}\right]\right|_{L^{2}(\Gamma)}\right)$

Proof. To bound the term $\left\langle\lambda_{h}-\lambda_{I}, \boldsymbol{n} \cdot\left[\boldsymbol{W}-\boldsymbol{W}_{I}\right]\right\rangle_{\Gamma}$, we first apply the Schwarz inequality to get

$$
\left\langle\lambda_{h}-\lambda_{I}, \boldsymbol{n} \cdot\left[\boldsymbol{W}-\boldsymbol{W}_{I}\right]\right\rangle_{\Gamma} \leq\left|\lambda_{h}-\lambda_{I}\right|_{L^{2}(\Gamma)}\left|\boldsymbol{W}-\boldsymbol{W}_{I}\right|_{L^{2}(\Gamma)},
$$

and then proceed as follows to bound the term $\left|\lambda_{h}-\lambda_{I}\right|_{L^{2}(\Gamma)}$. Since

$$
\left\langle\lambda_{h}, \boldsymbol{n} \cdot \boldsymbol{Y}\right\rangle_{\Gamma}=\left\langle\nabla_{\Gamma} \boldsymbol{W}_{h}, \nabla_{\Gamma} \boldsymbol{Y}\right\rangle_{\Gamma}, \quad \boldsymbol{Y} \in \boldsymbol{\Upsilon}_{h}, \quad\langle\lambda, \boldsymbol{n} \cdot \boldsymbol{Y}\rangle_{\Gamma}=\left\langle\nabla_{\Gamma} \boldsymbol{W}, \nabla_{\Gamma} \boldsymbol{Y}\right\rangle_{\Gamma}, \quad \boldsymbol{Y} \in \boldsymbol{H}^{1}(\Gamma)
$$

we can subtract the second equation from the first to obtain the error equation:

$$
\left\langle\lambda_{h}-\lambda_{I}, \boldsymbol{n} \cdot \boldsymbol{Y}\right\rangle_{\Gamma}=\left\langle\lambda-\lambda_{I}, \boldsymbol{n} \cdot \boldsymbol{Y}\right\rangle_{\Gamma}+\left\langle\nabla_{\Gamma}\left[\boldsymbol{W}_{h}-\boldsymbol{W}_{I}\right], \nabla_{\Gamma} \boldsymbol{Y}\right\rangle_{\Gamma}+\left\langle\nabla_{\Gamma}\left[\boldsymbol{W}_{I}-\boldsymbol{W}\right], \nabla_{\Gamma} \boldsymbol{Y}\right\rangle_{\Gamma}, \quad \boldsymbol{Y} \in \boldsymbol{\Upsilon}_{h} .
$$

Choose

$$
\boldsymbol{Y}=\sum_{i=1}^{N_{S}} \frac{\boldsymbol{n}_{i}^{+}+\boldsymbol{n}_{i}^{-}}{1+\boldsymbol{n}_{i}^{+} \cdot \boldsymbol{n}_{i}^{-}}\left[\lambda_{h}-\lambda_{I}\right]\left(\boldsymbol{x}_{i}\right) \phi_{i} .
$$

Note that $\boldsymbol{Y} \cdot \boldsymbol{n}$ will be a piecewise linear function. In fact, $\boldsymbol{Y} \cdot \boldsymbol{n}$ is also continuous, as we show below.

$$
\begin{aligned}
\boldsymbol{Y}\left(\boldsymbol{x}_{k}^{-}\right) \cdot \boldsymbol{n}\left(\boldsymbol{x}_{k}^{-}\right)=\boldsymbol{Y}\left(\boldsymbol{x}_{k}^{-}\right) \cdot \boldsymbol{n}_{k}^{-} & =\sum_{i=1}^{N_{S}} \frac{\boldsymbol{n}_{k}^{-} \cdot\left[\boldsymbol{n}_{i}^{+}+\boldsymbol{n}_{i}^{-}\right]}{1+\boldsymbol{n}_{i}^{+} \cdot \boldsymbol{n}_{i}^{-}}\left[\lambda_{h}-\lambda_{I}\right]\left(\boldsymbol{x}_{i}\right) \phi_{i}\left(\boldsymbol{x}_{k}^{-}\right) \\
& =\frac{\boldsymbol{n}_{k}^{-} \cdot\left[\boldsymbol{n}_{k}^{+}+\boldsymbol{n}_{k}^{-}\right]}{1+\boldsymbol{n}_{k}^{+} \cdot \boldsymbol{n}_{k}^{-}}\left[\lambda_{h}-\lambda_{I}\right]\left(\boldsymbol{x}_{k}\right)=\left[\lambda_{h}-\lambda_{I}\right]\left(\boldsymbol{x}_{k}\right), \\
\boldsymbol{Y}\left(\boldsymbol{x}_{k}^{+}\right) \cdot \boldsymbol{n}\left(\boldsymbol{x}_{k}^{+}\right)=\boldsymbol{Y}\left(\boldsymbol{x}_{k}^{+}\right) \cdot \boldsymbol{n}_{k}^{+} & =\sum_{i=1}^{N_{S}} \frac{\boldsymbol{n}_{k}^{+} \cdot\left[\boldsymbol{n}_{i}^{+}+\boldsymbol{n}_{i}^{-}\right]}{1+\boldsymbol{n}_{i}^{+} \cdot \boldsymbol{n}_{i}^{-}}\left[\lambda_{h}-\lambda_{I}\right]\left(\boldsymbol{x}_{i}\right) \phi_{i}\left(\boldsymbol{x}_{k}^{+}\right) \\
& =\frac{\boldsymbol{n}_{k}^{+} \cdot\left[\boldsymbol{n}_{k}^{+}+\boldsymbol{n}_{k}^{-}\right]}{1+\boldsymbol{n}_{k}^{+} \cdot \boldsymbol{n}_{k}^{-}}\left[\lambda_{h}-\lambda_{I}\right]\left(\boldsymbol{x}_{k}\right)=\left[\lambda_{h}-\lambda_{I}\right]\left(\boldsymbol{x}_{k}\right) .
\end{aligned}
$$


Hence, we see that $\boldsymbol{Y} \cdot \boldsymbol{n}$ is a continuous, piecewise, linear function satisfying $(\boldsymbol{Y} \cdot \boldsymbol{n})\left(\boldsymbol{x}_{k}\right)=\left(\lambda_{h}-\lambda_{I}\right)\left(\boldsymbol{x}_{k}\right)$, and so $\boldsymbol{Y} \cdot \boldsymbol{n}=\lambda_{h}-\lambda_{I}$. Now for $\mu \in \Lambda_{h}$,

$$
C_{1}|\mu|_{L^{2}(\Gamma)} \leq\left[h \sum_{i=1}^{N_{S}} \mu^{2}\left(\boldsymbol{x}_{i}\right)\right]^{1 / 2} \leq C_{2}|\mu|_{L^{2}(\Gamma)} .
$$

It easily follows from this fact and standard inverse estimates that

$$
|\boldsymbol{Y}|_{L^{2}(\Gamma)} \leq C\left|\lambda_{h}-\lambda_{I}\right|_{L^{2}(\Gamma)}, \quad\left|\nabla_{\Gamma} \boldsymbol{Y}\right|_{L^{2}(\Gamma)} \leq C h^{-1}\left|\lambda_{h}-\lambda_{I}\right|_{L^{2}(\Gamma)} .
$$

Using these results and applying the Schwarz inequality, we get

$$
\begin{aligned}
&\left|\lambda_{h}-\lambda_{I}\right|_{L^{2}(\Gamma)}^{2}=\left\langle\lambda_{h}-\lambda_{I}, \boldsymbol{n} \cdot \boldsymbol{Y}\right\rangle_{\Gamma}=\left\langle\lambda-\lambda_{I}, \lambda_{h}-\lambda_{I}\right\rangle_{\Gamma} \\
& \quad+\left\langle\nabla_{\Gamma}\left[\boldsymbol{W}_{h}-\boldsymbol{W}_{I}\right], \nabla_{\Gamma} \boldsymbol{Y}\right\rangle_{\Gamma}+\left\langle\nabla_{\Gamma}\left[\boldsymbol{W}_{I}-\boldsymbol{W}\right], \nabla_{\Gamma} \boldsymbol{Y}\right\rangle_{\Gamma}, \\
& \leq C\left(\left|\lambda-\lambda_{I}\right|_{L^{2}(\Gamma)}+h^{-1}\left|\nabla_{\Gamma}\left[\boldsymbol{W}_{h}-\boldsymbol{W}_{I}\right]\right|_{L^{2}(\Gamma)}+h^{-1}\left|\nabla_{\Gamma}\left[\boldsymbol{W}_{I}-\boldsymbol{W}\right]\right|_{L^{2}(\Gamma)}\right)\left|\lambda_{h}-\lambda_{I}\right|_{L^{2}(\Gamma)},
\end{aligned}
$$

and so

$$
\left|\lambda_{h}-\lambda_{I}\right|_{L^{2}(\Gamma)} \leq C\left(\left|\lambda-\lambda_{I}\right|_{L^{2}(\Gamma)}+h^{-1}\left|\nabla_{\Gamma}\left[\boldsymbol{W}_{h}-\boldsymbol{W}_{I}\right]\right|_{L^{2}(\Gamma)}+h^{-1}\left|\nabla_{\Gamma}\left[\boldsymbol{W}_{I}-\boldsymbol{W}\right]\right|_{L^{2}(\Gamma)}\right) .
$$

Hence,

$$
\begin{aligned}
&\left\langle\lambda_{h}-\lambda_{I}, \boldsymbol{n} \cdot\left[\boldsymbol{W}-\boldsymbol{W}_{I}\right]\right\rangle_{\Gamma} \leq\left|\lambda_{h}-\lambda_{I}\right|_{L^{2}(\Gamma)}\left|\boldsymbol{W}-\boldsymbol{W}_{I}\right|_{L^{2}(\Gamma)} \\
& \leq C\left|\boldsymbol{W}-\boldsymbol{W}_{I}\right|_{L^{2}(\Gamma)}\left(\left|\lambda-\lambda_{I}\right|_{L^{2}(\Gamma)}+h^{-1}\left|\nabla_{\Gamma}\left[\boldsymbol{W}_{h}-\boldsymbol{W}_{I}\right]\right|_{L^{2}(\Gamma)}+h^{-1}\left|\nabla_{\Gamma}\left[\boldsymbol{W}_{I}-\boldsymbol{W}\right]\right|_{L^{2}(\Gamma)}\right) .
\end{aligned}
$$

Remarks. The constants that appear in the error estimates depend (in general) on the curvature of $\Gamma$ (the true boundary). Therefore, an a priori estimate on the curvature of the time-dependent domain boundary is necessary to proceed to a full time-dependent analysis.

\section{Solving the Mixed System}

We describe here a simple iterative (Uzawa) method for computing $\lambda$ with a predetermined step size $\rho$. More sophisticated methods (e.g., conjugate gradient Uzawa with exact line searches) follow similar lines (see [17]). All the remaining variables are then easily computed as functions of $\lambda$.

7.1. Uzawa Method. We define a stopping tolerance: $\epsilon>0$, step size $\rho>0$ sufficiently small, and proceed as follows.

(1) Let $\lambda^{0} \in \Lambda_{h}$ (initial guess).

(2) $\mathbf{F O R} k=0,1,2, \ldots$

(3) Solve: find $\boldsymbol{u}^{k} \in \boldsymbol{V}_{h}, p^{k} \in Q_{h}$ such that

$$
\begin{aligned}
\left(\frac{\alpha}{\Delta t}+\beta\right)\left(\boldsymbol{u}^{k}, \boldsymbol{v}\right)_{\Omega}- & \left(p^{k}, \operatorname{div} \boldsymbol{v}\right)_{\Omega}=\frac{\alpha}{\Delta t}(\boldsymbol{U}, \boldsymbol{v})_{\Omega}-\langle E, \boldsymbol{v} \cdot \boldsymbol{n}\rangle_{\Gamma}-\left\langle\lambda^{k}, \boldsymbol{v} \cdot \boldsymbol{n}\right\rangle_{\Gamma}, \quad \forall \boldsymbol{v} \in \boldsymbol{V}_{h} \\
\left(q, \operatorname{div} \boldsymbol{u}_{k}\right)_{\Omega} & =0, \quad \forall q \in Q_{h}
\end{aligned}
$$

and find $\boldsymbol{W}^{k} \in \boldsymbol{\Upsilon}_{h}$ such that

$$
\begin{gathered}
\left\langle\nabla_{\Gamma} \boldsymbol{W}^{k}, \nabla_{\Gamma} \boldsymbol{Y}\right\rangle_{\Gamma}=\left\langle\lambda^{k}, \boldsymbol{n} \cdot \boldsymbol{Y}\right\rangle_{\Gamma}, \quad \forall \boldsymbol{Y} \in \boldsymbol{\Upsilon}_{h}, \\
\frac{1}{\Delta t}\left\langle\boldsymbol{W}^{k} \cdot \boldsymbol{n}, \phi\right\rangle_{\Gamma}-\left\langle\boldsymbol{u}^{k} \cdot \boldsymbol{n}, \phi\right\rangle_{\Gamma}=\frac{1}{\Delta t}\left\langle\operatorname{id}_{\Gamma} \cdot \boldsymbol{n}, \phi\right\rangle_{\Gamma}, \quad \phi=x, y . \quad \leftarrow \quad \text { (set constant part.) }
\end{gathered}
$$

(4) Update $\lambda^{k+1} \in \Lambda_{h}$ by

$$
\lambda^{k+1}=\lambda^{k}-\rho \Pi\left(\frac{1}{\Delta t} \boldsymbol{W}^{k} \cdot \boldsymbol{n}-\boldsymbol{u}^{k} \cdot \boldsymbol{n}-\frac{1}{\Delta t} \mathrm{id}_{\Gamma} \cdot \boldsymbol{n}\right),
$$

where $\Pi$ denotes the $L^{2}(\Gamma)$ projection into $\Lambda_{h}$. 
(5) If $\left\|\lambda^{k+1}\right\|_{L^{2}(\Gamma)} /\left\|\lambda^{0}\right\|_{L^{2}(\Gamma)} \leq \epsilon$, then set $\lambda=\lambda^{k+1}$ and EXIT LOOP.

(6) END FOR.

\section{Numerical Computations}

In this final section, we show two simulations of the EWOD model using the algorithm proposed in this paper. Figure 1 shows a simulation of the EWOD model in which the electrowetting force term is defined via an auxiliary function $\widehat{E}$ that is time-independent and defined over $[0,1] \times[0,1]$ (unit square). Then $E$ is defined (i.e., the term appearing in the pressure boundary condition) as the restriction of $\widehat{E}$ to $\Gamma$. We chose $\widehat{E}$ in order to drive the five droplets toward each other. Once the droplets are sufficiently close, we stop the simulation and use a hybrid level set/mesh generation method to enable the topological change [29, 39]; this happens during one time-step and creates a new domain $\widetilde{\Omega}$. We then restart the simulation using $\widetilde{\Omega}$ as the initial domain with initial velocity obtained by interpolating from the previous domain. After the droplets merge, the force $E$ is set to zero. Thus, the remainder of the evolution is only governed by surface tension, which causes the large (merged) droplet to settle into a circular shape.

Figure 2 shows a simulation of droplet splitting by EWOD. The auxiliary function $\widehat{E}$ is chosen to be negative on the left and right regions of the unit square, zero in the middle, and continuous everywhere. This mimics the actuation used in some EWOD devices; see [43] for an example. Thus, just as before, $E$ is obtained by restricting $\widehat{E}$ to $\Gamma$. The droplet splits into two large pieces and one small satellite droplet. After the split, we set $E$ to zero which causes the droplets to relax to a circular shape. We use the same hybrid level set/mesh generation method to enable the droplet to split.

\section{REFERENCES}

1. Helmut Abels, Harald Garcke, and Günther Grün, Thermodynamically consistent, frame indifferent diffuse interface models for incompressible two-phase flows with different densities, Mathematical Models and Methods in Applied Sciences (M3AS) 22 (2012), no. 3.

2. Eberhard Bänsch, Finite element discretization of the navier-stokes equations with a free capillary surface, Numerische Mathematik 88 (2001), 203-235.

3. A. S. Bedekar, J. W. Jenkins, and S. Sundaram, A computational model for the design of electrowetting on dielectric (ewod) systems, NSTI Nanotechnology Conference \& Trade Show (Anaheim, California), vol. Session Presentation, May 2005.

4. B. Berge, Électrocapillarité et mouillage de films isolants par l'eau (including an english translation), Comptes Rendus de l'Académie des Sciences de Paris, Série II 317 (1993), 157-163.

5. B. Berge and J. Peseux, Variable focal lens controlled by an external voltage: An application of electrowetting, European Physical Journal E 3 (2000), no. 2, 159-163.

6. J. Bergh and J. Löfström, Interpolation spaces. An introduction, Springer-Verlag, Berlin, 1976, Grundlehren der Mathematischen Wissenschaften, No. 223.

7. K. Brakke, The surface evolver, Experimental Mathematics 1 (1992), no. 2, 141-165.

8. F. Brezzi and M. Fortin, Mixed and hybrid finite element methods, Springer-Verlag, New York, NY, 1991.

9. A.-P. Calderón, Intermediate spaces and interpolation, the complex method, Studia Math. 24 (1964), 113-190.

10. Sung Kwon Cho and Chang-Jin Kim, Particle separation and concentration control for digital microfluidic systems, The 16th Annual IEEE International Conference on MEMS (Kyoto, Japan), January 2003, pp. 686-689.

11. Sung Kwon Cho, Hyejin Moon, and Chang-Jin Kim, Creating, transporting, cutting, and merging liquid droplets by electrowetting-based actuation for digital microfluidic circuits, Journal of Microelectromechanical Systems 12 (2003), no. 1, 70-80.

12. Philippe G. Ciarlet, The finite element method for elliptic problems, 2nd ed., Classics in Applied Mathematics, SIAM, Philadelphia, PA, 2002, ISBN: 978-0898715149.

13. Michel C. Delfour and Jean-Paul Zolésio, Shapes and geometries: Analysis, differential calculus, and optimization, Advances in Design and Control, vol. 4, SIAM, 2001. 


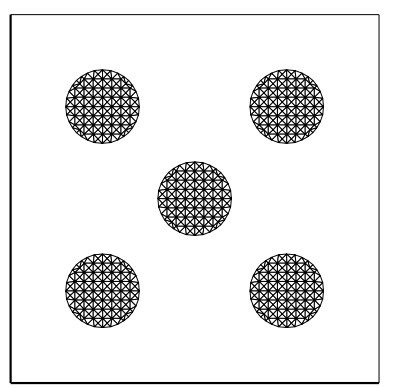

Time: 0.00000

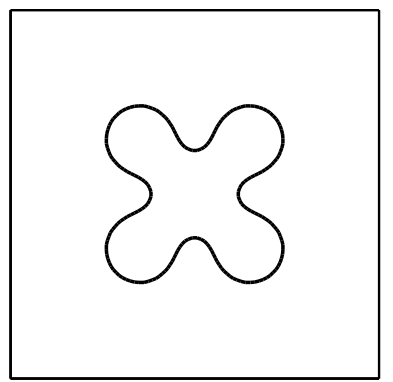

Time: 1.50831

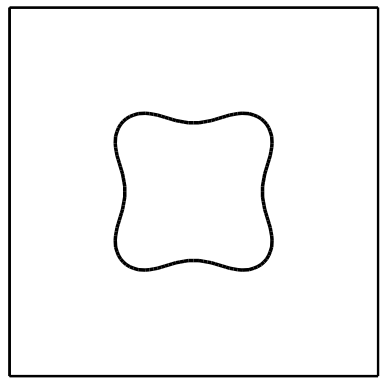

Time: 1.53623

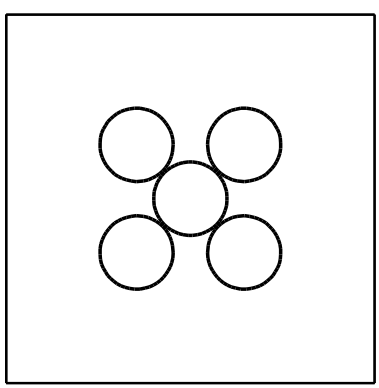

Time: 1.49630

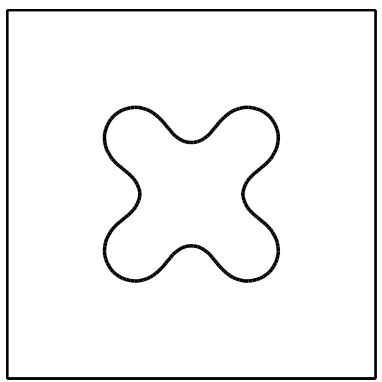

Time: 1.51351

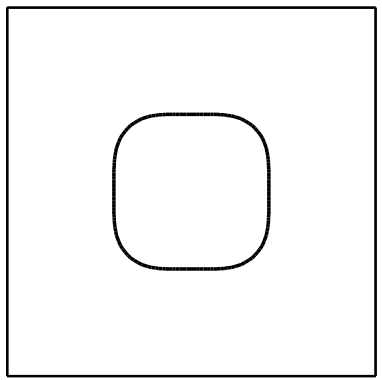

Time: 1.55656

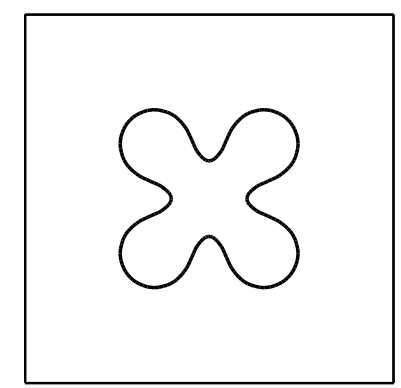

Time: 1.50630

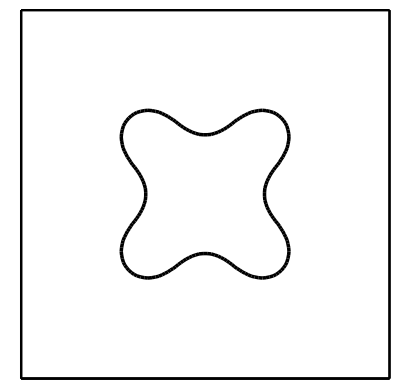

Time: 1.52132

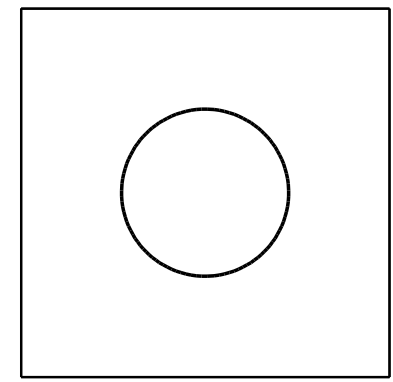

Time: 1.65814

Figure 1. Simulation of EWOD model, equations (5.1)-(5.4). Five droplets are pushed together to merge into a single droplet by an appropriately chosen $E$ (i.e., the electrowetting force term). The plot at time 0.0 shows the mesh of the fluid domain $\Omega$; subsequent plots only show the interface $\Gamma$.

14. C. Eck, M. Fontelos, G. Grün, F. Klingbeil, and O. Vantzos, On a phase-field model for electrowetting, Interfaces and Free Boundaries 11 (2009), no. 2, 259290.

15. L. C. Evans, Partial differential equations, American Mathematical Society, Providence, Rhode Island, 1998.

16. M. Fontelos, G. Grn, and S. Jrres, On a phase-field model for electrowetting and other electrokinetic phenomena, SIAM Journal on Mathematical Analysis 43 (2011), no. 1, 527-563.

17. Roland Glowinski, Numerical methods for fluids (part 3), vol. 9, Handbook of Numerical Analysis, no. 3, North-Holland, January 2003.

18. J. Gong, S. K. Fan, and C. J. Kim, Portable digital microfluidics platform with active but disposable lab-on-chip, 17th IEEE International Conference on Micro Electro Mechanical Systems (MEMS) (Maastricht, The Netherlands), IEEE Press, Jan 2004, ISBN: 0-7803-8265-x, pp. 355-358.

19. Robert A. Hayes and B. J. Feenstra, Video-speed electronic paper based on electrowetting, Nature 425 (2003), no. 6956, 383-385.

20. Junghoon Lee, Hyejin Moon, Jesse Fowler, Thomas Schoellhammer, and Chang-Jin Kim, Electrowetting and electrowettingon-dielectric for microscale liquid handling, Sensors and Actuators, A-Physics (95), 2002, pp. 259-268. 


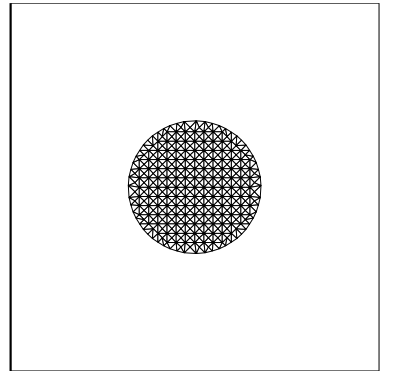

Time: 0.00000

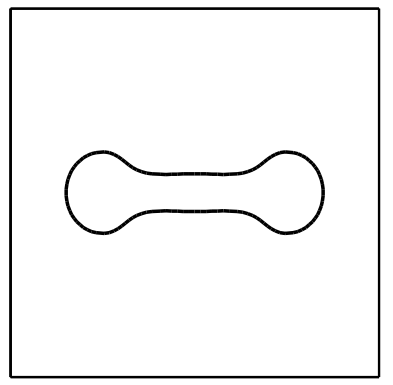

Time: 0.24000

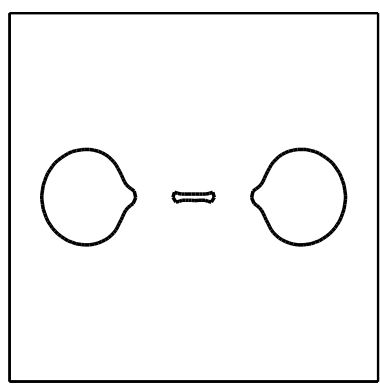

Time: 0.43150

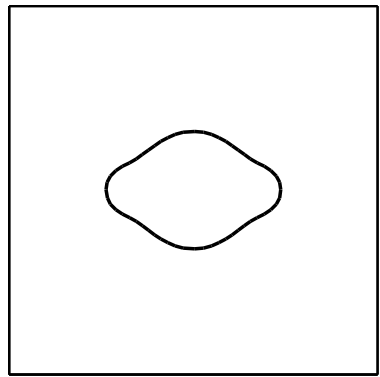

Time: 0.08000

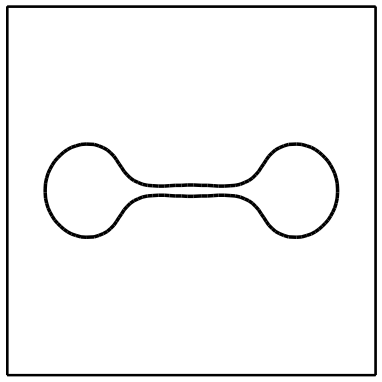

Time: 0.36800

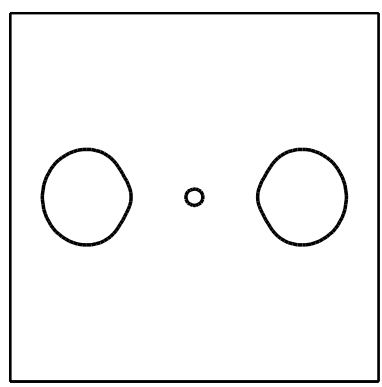

Time: 0.43267

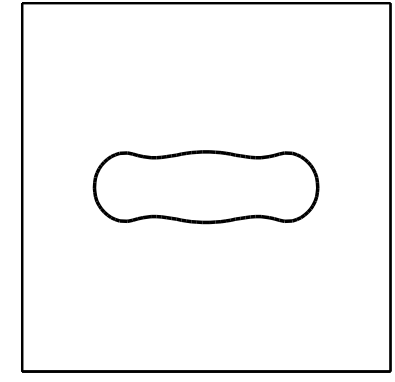

Time: 0.16000

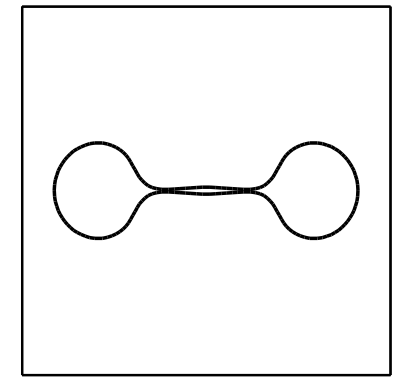

Time: 0.43100

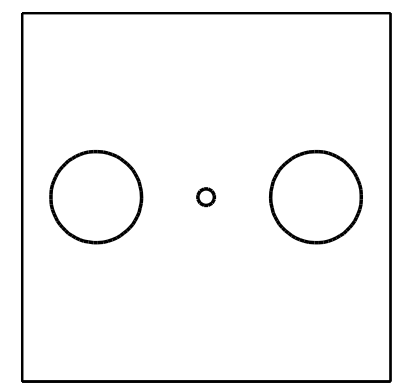

Time: 0.54617

Figure 2. Simulation of EWOD model, equations (5.1)-(5.4). Single droplet splitting due to a particular choice of $E$. The plot at time 0.0 shows the mesh of the fluid domain $\Omega$; subsequent plots only show the interface $\Gamma$.

21. J. Lienemann, A. Greiner, and J. G. Korvink, Modeling, simulation, and optimization of electrowetting, IEEE Transactions on Computer-Aided Design of Integrated Circuits and Systems 25 (2006), no. 2, 234-247.

22. H.-W. Lu, K. Glasner, A. L. Bertozzi, and Chang-Jin Kim, A diffuse interface model for electrowetting drops in a hele-shaw cell, Journal of Fluid Mechanics 590 (2007), 411 - 435.

23. L. Minnema, H. A. Barneveld, and P. D. Rinkel, An investigation into the mechanism of water treeing in polyethylene high voltage cables, IEEE Transactions on Electrical Insulators 15 (1980), 461-472.

24. Kamran Mohseni and Ali Dolatabadi, An electrowetting microvalve: Numerical simulation, Ann NY Acad Sci 1077 (2006), $415-425$.

25. Hyejin Moon, Aaron R. Wheeler, Robin L. Garrell, Joseph A. Loo, and Chang-Jin Kim, On-chip sample preparation by electrowetting-on-dielectric digital microfluidcs for matrix assisted laser desorption/ionization mass spectrometry, Proceedings of IEEE MEMS (Miami, Florida), Feb 2005, pp. 859-862.

26. Marwan Moubachir and Jean-Paul Zolésio, Moving shape analysis and control: Applications to fluid structure interactions, Pure and Applied Mathematics, vol. 277, Chapman and Hall/CRC, 2006.

27. F. Mugele and J.-C. Baret, Electrowetting: from basics to applications, Journal of Physics: Condensed Matter 17 (2005), R705-R774. 
28. Ricardo H. Nochetto, Abner J. Salgado, and Shawn W. Walker, A diffuse interface model for electrowettng with moving contact lines, submitted. (2012).

29. Ricardo H. Nochetto and Shawn W. Walker, A hybrid variational front tracking-level set mesh generator for problems exhibiting large deformations and topological changes, Journal of Computational Physics 229 (2010), no. 18, $6243-6269$.

30. Thibault Roques-Carmes, Robert A. Hayes, B. J. Feenstra, and L. J. M. Schlangen, Liquid behavior inside a reflective display pixel based on electrowetting, Journal of Applied Physics 95 (2004), no. 8, 4389-4396.

31. Thibault Roques-Carmes, Robert A. Hayes, and L. J. M. Schlangen, A physical model describing the electro-optic behavior of switchable optical elements based on electrowetting, Journal of Applied Physics 96 (2004), no. 11, 6267-6271.

32. F. Saeki, J. Baum, H. Moon, J.-Y. Yoon, C.-J. Kim, and R. L. Garrell, Electrowetting on dielectrics (ewod): Reducing voltage requirements for microfluidics, Polym. Mater. Sci. Eng. 85 (2001), 12-13.

33. W. Satoh, M. Loughran, and H. Suzuki, Microfluidic transport based on direct electrowetting, Journal of Applied Physics 96 (2004), no. 1, 835-841.

34. Benjamin Shapiro, Hyejin Moon, Robin Garrell, and Chang-Jin Kim, Equilibrium behavior of sessile drops under surface tension, applied external fields, and material variations, Journal of Applied Physics 93 (2003), no. 9, $5794-5811$.

35. Erwin Stein, Ren de Borst, and Thomas J.R. Hughes (eds.), Encyclopedia of computational mechanics: Vol. 1 - fundamentals, 1st ed., vol. 1, Wiley, 2004.

36. Roger M. Temam and Alain M. Miranville, Mathematical modeling in continuum mechanics, 2nd ed., Cambridge University Press, 2005.

37. Clifford A. Truesdell, A first course in rational continuum mechanics, Pure and applied mathematics, a series of monographs and textbooks, Academic Press, 1976.

38. Shawn W. Walker, Modeling, simulating, and controlling the fluid dynamics of electro-wetting on dielectric, Ph.D. thesis, University of Maryland, College Park, August 2007.

39. - Tetrahedralization of isosurfaces with guaranteed-quality by edge rearrangement (tiger), submitted (2012).

40. Shawn W. Walker, Andrea Bonito, and Ricardo H. Nochetto, Mixed finite element method for electrowetting on dielectric with contact line pinning, Interfaces and Free Boundaries 12 (2010), no. 1, 85-119.

41. Shawn W. Walker and Benjamin Shapiro, A control method for steering individual particles inside liquid droplets actuated by electrowetting, Lab on a Chip 5 (2005), 1404-1407.

42. _ Modeling the fluid dynamics of electrowetting on dielectric (ewod), Journal of Microelectromechanical Systems $\mathbf{1 5}$ (2006), no. 4, 986-1000.

43. Shawn W. Walker, Benjamin Shapiro, and Ricardo H. Nochetto, Electrowetting with contact line pinning: Computational modeling and comparisons with experiments, Physics of Fluids 21 (2009), no. 10, 102103.

44. Aaron R. Wheeler, Hyejin Moon, Chang-Jin Kim, Joseph A. Loo, and Robin L. Garrell, Electrowetting-based microfluidics for analysis of peptides and proteins by matrix-assisted laser desorption/ionization mass spectrometry, Analytical Chemistry 76 (2004), 4833-4838.

Department of Mathematics, Rutgers University, Piscataway, NJ 08854

E-mail address: falk@math.rutgers.edu

$U R L:$ http://www.math.rutgers.edu/ falk/

Department of Mathematics, Louisiana State University, Baton Rouge, LA 70803

E-mail address: walker@math.1su..edu

URL: http://www.math.lsu.edu/ walker/ 